Heirs of box types in polynomially bounded structures

Tressl, Marcus

2008

MIMS EPrint: 2008.87

Manchester Institute for Mathematical Sciences

School of Mathematics

The University of Manchester

\footnotetext{
Reports available from: http://eprints.maths.manchester.ac.uk/

And by contacting: The MIMS Secretary

School of Mathematics

The University of Manchester

Manchester, M13 9PL, UK
} 


\title{
Heirs of box types in polynomially bounded structures
}

\author{
Marcus Tressl
}

\begin{abstract}
A box type is an $n$-type of an o-minimal structure which is uniquely determined by the projections to the coordinate axes. We characterize heirs of box types of a polynomially bounded o-minimal structure $M$. From this, we deduce various structure theorems for subsets of $M^{k}$, definable in the expansion $\mathscr{M}$ of $M$ by all convex subsets of the line, e.g. we obtain model completeness of $\mathscr{M}$, provided $M$ is model complete.
\end{abstract}

\author{
Contents \\ 1. Introduction \\ 2. Heirs of cuts in polynomially bounded structures \\ 3. Box types \\ 4. The box type associated to a cut \\ 5. Two methods producing box types \\ 6. Heirs of box types \\ 7. Model completeness of exhaustive expansions \\ 8. Structure theorems for exhaustive expansions
}

\section{INTRODUCTION}

The notion "heir" of a type $p$ of a first order structure $M$ has been introduced by Lascar and Poizat (cf. [La-Po], §3) in order to define Shelah's forking in stable theories (cf. [She1]). An heir of $p$ on some elementary extension $N$ of $M$ is a type $q$ of $N$ such that for every formula $\varphi(\bar{x}, \bar{y})$ with parameters from $M$ and every $\bar{y}$-tuple $\bar{n} \subseteq N$, if $\varphi(\bar{x}, \bar{n}) \in q$, then there is some $\bar{m} \subseteq M$ such that $\varphi(\bar{x}, \bar{m}) \in p$. Geometrically, an heir $q$ of $p$ is a generic extension of $p$ on $N$, since - by definition - $q$ does not introduce more relations among a realization of $q$ than $p$ already knows. A compactness argument shows that $p$ always has an heir on $N$.

For example let $T=A C F$ be the theory of algebraically closed fields. By quantifier elimination, a type $p$ of $M=A C F$ is naturally viewed as a prime ideal $\mathfrak{p}$ of the polynomial ring $M[\bar{X}]$ and $p$ is the generic point of the variety $V$ defined by the polynomials from $\mathfrak{p}$. In this case, $q$ is an heir of $p$ if and only if $q$ is the generic point of $V \times_{M} N$ (cf. [La], section 4.5). The same characterization holds for the theory of differentially closed fields.

An important place where heirs appear, is the characterization of definability of a type, which says that $p$ is definable if and only if $p$ has a unique heir on every elementary extension of $M$ (cf. [La-Po], Theorem 4.2). Recall that a type $p$ of a structure $M$ is called definable if for every formula $\varphi(\bar{x}, \bar{y})$, the set of all $\bar{y}$-tuples $\bar{m} \subseteq M$ with $\varphi(\bar{x}, \bar{m}) \in p$ is parametrically definable in $M$. Intuitively this says that the membership problem for $p$ is solvable, e.g. if $p$ "is" a prime ideal as explained above, then definability precisely says that the membership problem for the ideal $\mathfrak{p}$ is solvable.

2000 Mathematics Subject Classification: Primary 03C64; Secondary 13J30

Keywords and phrases: model theory, o-minimality, real closed fields, heirs, weakly ominimal, model completeness, Dedekind cuts, valuation theory 
For o-minimal theories, less is known about an algebraic description of heirs of types. Partial description may be found at several places:

Firstly, heirs are related to the Marker-Steinhorn Theorem (cf. [Ma-St]), which says that a type $p$ of an o-minimal structure $M$ is definable if and only if it is tame, i.e. $M$ is Dedekind complete in $M\langle\bar{\alpha}\rangle$, where $\bar{\alpha}=p$. In [Tr4] this theorem is re-proved by applying the definability criterion above. It should also be mentioned here that an $n$-type $p$ of a real closed field $R$ is definable if and only if the ordering $\{f \in R[\bar{X}] \mid f \geq 0 \in p\}$ of $R[\bar{X}]$ is a weakly semi-algebraic subset of $R[\bar{X}]$ in the sense of $[\mathrm{Kn}]$. Due to the definability criterion, a possible characterization of an heir of a tame type $p$ will take place inside tame types and we know that there is only one heir of a tame type. This is a dramatic simplification of the question how to characterize heirs of tame types, but it does not solve it.

A second place which deals implicitly with heirs, is the work of Dolich (cf. [Do]), where forking of sets is analyzed. It is unclear how this may be used to characterize heirs of types.

Certainly the terms "characterization of heirs" and "algebraic description of heirs" is vague. What we have in mind is: find invariants of the types which witness heritage, or - in the case of real closed fields - find constructions, possibly from (real) commutative algebra which produce all heirs of orderings of a polynomial ring over a real closed field on a given real closed over-field.

In the case of 1-types of polynomially bounded structures, a complete description of heirs in terms of invariance groups and invariance rings is proved in [Tr1] (this is explained and summarized in detail below in (2.16)(i),(ii),(iii)). Recall that the invariance group of a 1-type $p$ (or a cut $p$ ) of $M$ is the convex group $G(p):=\{a \in M \mid a+p=p\}$ and the invariance ring of $p$ is the convex valuation ring $\{b \in M \mid b \cdot G(p) \subseteq G(p)\}$. Observe that these invariants are formulated inside the underlying real closed field. Still, in the case of 1-types of pure real closed fields, a characterization in terms of real commutative algebra is missing.

In each context where heirs appear above, the results reveal significant structure theorems about the o-minimal theory under examination.

In this paper we characterize heirs of types over real closed fields - more generally over polynomially bounded, o-minimal structures $M$ for so called "box types". A box type of $M$ is an $n$-type $p$ of $M$ of dimension $n$ such that $p$ is uniquely determined by the projections of $p$ to the coordinate axes; one might also say that these projections are 'orthogonal'. Certainly many $n$-types of an o-minimal field are not box types, e.g. if $p=p(x, y)$ is a 2-type and the projection $p_{x}$ of $p$ to the $x$-axis is not realized and equal to the projection $p_{y}$ of $p$ to the $y$-axis; then there are at least two 2-types of dimension 2 which have the same projection, separated by the formula $x<y$. More general, if $p$ is a 2-type of dimension 2 such that $p_{x}$ and $p_{y}$ are definable, then there is an $M$-definable function $f$ with $f\left(p_{x}\right)=p_{y}$ and there are at least two 2-types of dimension 2 which have projections $p_{x}, p_{y}$, separated by the formula $f(x)<y$. This argument can easily be extended to show that a definable type of dimension $n$ is a box type if and only if $n=1$.

Here are some examples of box types, which are denoted in the following way: we state a sequence of 1-types (or Dedekind cuts) and each of these sequences will determine a unique $n$-type. We'll work in pure real closed fields. Let $\mathbb{R}_{\text {alg }}$ denote the field of real algebraic numbers.

a. Let $r_{1}, \ldots, r_{n} \in \mathbb{R}$ be algebraically independent over $\mathbb{Q}$. Then

$$
\left(t p\left(r_{1} / \mathbb{R}_{\text {alg }}\right), \ldots, t p\left(r_{n} / \mathbb{R}_{\text {alg }}\right)\right)
$$

is a box type.

b. Let $R \subseteq S$ be real closed fields and let $x_{1}, \ldots, x_{n} \in S \backslash R$ such that for each $i$, the set 
$V_{i}:=\left\{a \in R|| a \mid<x_{i}\right\}$ is a convex valuation ring of $R$. If the $V_{i}$ are mutually distinct, then $\left(t p\left(x_{1} / R\right), \ldots, t p\left(x_{n} / R\right)\right)$ is a box type.

c. Let $S:=\mathbb{R}\left(\left(t^{\mathbb{R}}\right)\right)$ be the formal power series field over $\mathbb{R}$ with value group $\mathbb{R}$ and let $R$ be the real closure of $\mathbb{R}_{\text {alg }}(t)$ in $S$. Let $x:=\sum \frac{t^{k}}{k !}$. Then $\left(t p(\pi / R), t p(x / R), t p\left(t^{\pi} / R\right)\right)$ is a box type.

That these examples are box types basically follows from valuation theory. In section 5 we present two methods to produce box types: A consequence of Theorem (5.1) for example says that $\left(p_{1}, \ldots, p_{n}\right)$ is a box type whenever the $p_{i}$ have distinct invariance rings; (5.4) is a criterion to detect box types which does not (necessarily) refer to distinct invariance rings.

By an heir of a box type $\left(p_{1}, \ldots, p_{n}\right)$ we mean a sequence $\left(q_{1}, \ldots, q_{n}\right)$ of 1 -types extending the given one, such that the structure $M$ expanded by the sets $p_{i}^{L}:=\left\{a \in M \mid a<p_{i}\right\}$ is existentially closed in $\left(N, q_{1}^{L}, \ldots, q_{n}^{L}\right)$. Then, if $\left(q_{1}, \ldots, q_{n}\right)$ is again a box type, this is precisely the model theoretic notion described at the beginning. Notice that in this case and if $q_{i}$ is an heir of $p_{i}$ for each $i$ then $\left(q_{1}, \ldots, q_{n}\right)$ is in general not an heir of $\left(p_{1}, \ldots, p_{n}\right)$ : an explanation can be found in the text after (2.5), a concrete example can be found in (4.3). Again, the case of distinct invariance rings is an exceptionally friendly case here: $\mathrm{By}(6.2)$, if the $p_{i}$ have distinct invariance rings then choosing heirs $q_{i}$ of $p_{i}$ produces an heir $\left(q_{1}, \ldots, q_{n}\right)$ of $\left(p_{1}, \ldots, p_{n}\right)$.

Our main result is theorem (6.6), which is of technical nature. The theorem - in principal - characterizes the heirs $\left(q_{1}, \ldots, q_{n}\right)$ of a box type $\left(p_{1}, \ldots, p_{n}\right)$ in terms of the location of the invariance groups and the invariance rings of the $q_{i}$ compared with the location of those of the $p_{i}$. "In principal" here means that we actually need an assumption on $\left(p_{1}, \ldots, p_{n}\right)$ concerning the location of the invariance groups and rings of the $p_{i}$; but for every box type $\left(r_{1}, \ldots, r_{k}\right)$, there is a box type $\left(p_{1}, \ldots, p_{n}\right)$ satisfying this assumption such that $\left(M, r_{1}^{L}, \ldots, r_{k}^{L}\right)$ is parametrically interdefinable with $\left(M, p_{1}^{L}, \ldots, p_{n}^{L}\right)$ (cf. (7.6)).

Theorem (6.6) has various consequences. Firstly, the Robinson test implies a model completeness result (cf. (7.4)) of model complete, polynomially bounded structures expanded by finitely many convex subsets. This for example can be used to show that for each real closed field $R$ there is a set $\mathbb{D}$ of convex subsets of $R$ such that $R$ in the language of rings expanded by all sets from $\mathbb{D}$ is model complete and such that every convex subset of $R$ is parametrically definable in the expanded structure (cf. (7.7)).

In order to describe further consequences, let $\mathscr{M}$ denote the expansion of a polynomially bounded structure $M$ by $n$ convex subsets of $M$. Then

1. There is an elementary extension $N \succ M$ with $\operatorname{dim} N / M \leq 6 n$ such that every subset of $M^{k}$, definable in $\mathscr{M}$ is of the form $Z \cap M^{k}$, for some $Z \subseteq N^{k}$, definable in $N$ (cf. (8.2)). Moreover the bound $6 n$ is sharp in general (cf. (8.3)).

2. For every function $f: M^{k} \longrightarrow M^{r}$, definable in $\mathscr{M}$, there is a finite partition $X_{1} \cup \ldots \cup X_{m}$ of $M^{k}$ into $\mathscr{M}$-definable sets, such that $\left.f\right|_{X_{i}}$ is the restriction to $X_{i}$ of an $M$-definable function $M^{k} \longrightarrow M^{r}$ (cf. (8.5)).

3 . If there is an $\mathscr{M}$-definable subset of $M$, which can not be defined in any $(M, V)$ for a convex valuation ring $V$ of $M$, then $\mathscr{M}$ does not have definable Skolem functions (cf. $(8.6))$.

4. The curve selection lemma holds in $\mathscr{M}$ (cf. (8.7)).

Finally, theorem (8.8) describes the subsets of $M$, which are definable in $\mathscr{M}$.

These results extend the structure theory of weakly o-minimal structures (cf. [MMS], [We1], [We2]), for our expansion $\mathscr{M}$. 
Notice that application 1 above without the dimension bound is already known from [BaPo]: It is shown there, that an o-minimal structure expanded by all trace sets, i.e. sets which are intersections of $M^{n}$ with some set definable in an elementary extension of $M$, has quantifier elimination in the language which has a predicate for each trace set. It is an open question whether every trace set is already definable in the structure $M$ expanded by all convex subsets of the line.

\section{Heirs of CUtS in POLYNOMially BOUNDED STRUCTURES}

In this section we recall notions and results on cuts of o-minimal structures, mainly from $[\operatorname{Tr} 1]$ and $[\operatorname{Tr} 2]$. Basic algebra of cuts in ordered abelian groups and o-minimal expansions of groups can be found in sections 2 and 3 of [Tr1].

Let $X$ be a totally ordered set. A (Dedekind) cut $p$ of $X$ is a tuple $\left(p^{L}, p^{R}\right)$ of subsets $p^{L}, p^{R}$ of $X$ such that $p^{L} \cup p^{R}=X$ and $p^{L}<p^{R}$, i.e. $a<b$ for all $a \in p^{L}, b \in p^{R} . p^{L}$ and $p^{R}$ are called the left and the right options of the cut $p$, respectively. If $Y \subseteq X$, then $Y^{+}$ denotes the cut $p$ of $X$ with $p^{R}=\{x \in X \mid x>Y\}$. $Y^{+}$is called the upper edge of $Y$. Similarly the lower edge $Y^{-}$of $Y$ is defined. A cut $p$ of $X$ is called principal if $p^{L}$ has a supremum in $X \cup\{ \pm \infty\}$, in other words if and only if $p$ is of the form $+\infty,-\infty$ or of the form $a^{+}, a^{-}$for some $a \in X$. A cut $q$ of an ordered set containing $X$ extends a cut $p$ of $X$ if $q^{L} \cap X=p^{L}$.

Recall that the set $X \cup\{$ the cuts of $X\}$ is totally ordered in the obvious way and Dedekind complete (it is not the Dedekind completion, since the principal cuts different from $\pm \infty$ are not in the Dedekind completion). If $Y \supseteq X$ is an extension of totally ordered sets and $p$ is a cut of $X$ then there is a least and a largest cut of $Y$ extending $p$.

Let $p$ be a cut of an ordered abelian group $K$. The convex subgroup

$$
G(p):=\{a \in K \mid a+p=p\}
$$

of $K$ is called the invariance group of $p$ (here $a+p:=\left(a+p^{L}, a+p^{R}\right)$ ). The cut $G(p)^{+}$is denoted by $\hat{p}$. Notice that $G(p)=G(p+a)$ for every $a \in K$, in particular for every convex subgroup $U$ of $K$ we have $G\left(a+U^{+}\right)=G\left(U^{+}\right)=U=G\left(U^{-}\right)=G\left(a+U^{-}\right)$.

If $K$ is an ordered field, then $G(p)$ denotes the invariance group of $p$ with respect to $(K,+, \leq)$ and $G^{*}(p)$ denotes the invariance group of $|p|(=\max \{p,-p\})$ with respect to $\left(K^{>0}, \cdot, \leq\right)$; thus $G^{*}(p)=\{a \in K \mid a \cdot p=p\}$. If $G$ is a convex subgroup of $(K,+, \leq)$, then the convex valuation ring

$$
V(G):=\{a \in K \mid a \cdot G \subseteq G\}
$$

of $K$ is called the invariance ring of $G$. If $p$ is a cut of $K$, then $V(p):=V(G(p))$ is called the invariance ring of $p$. Note that the group of positive units of $V(p)$ is the multiplicative invariance group of $\hat{p}$. The maximal ideal of $V(p)$ is denoted by $\mathfrak{m}(p)$.

Note also that $G(a \cdot p)=a \cdot G(p)=\{a \cdot g \mid g \in G(p)\}$ for every cut $p$ of $K$ and every $a \in K^{*}$; here $a \cdot p=\left(a \cdot p^{L}, a \cdot p^{R}\right)$ if $a>0$ and $a \cdot p=-(-a \cdot p)$ if $a<0$.

By [Tr1], (3.5), if $p>\hat{p}$, then there is some $c \in K$ such that

$$
G^{*}(p)=c \cdot G(p)+1(=\{c \cdot a+1 \mid a \in G(p)\}) .
$$


(2.1) Definition. Let $K$ be a divisible ordered abelian group and let $p$ be a cut of $K$. We define the signature of $p$ as

$$
\operatorname{sign} p:= \begin{cases}1 & \text { if there is a convex subgroup } G \text { of } K \text { and some } a \in K \text { with } p=a+G^{+} \\ -1 & \text { if there is a convex subgroup } G \text { of } K \text { and some } a \in K \text { with } p=a-G^{+} \\ 0 & \text { otherwise. }\end{cases}
$$

Since $K$ is divisible we can not have $a+G^{+}=b-H^{+}$for $a, b \in K$ and convex subgroups $G, H$ of $K$. Hence the signature is well defined. If $K$ is a real closed field, then $\operatorname{sign}^{*} p$ denotes the signature of $|p|$ with respect to $\left(K^{>0}, \cdot, \leq\right)$.

In the sequel we shall work with o-minimal structures and theories. We refer to [PS] and to section 1 of [vdD-Lew] for an introduction to the subject. In the moment we work with a complete, o-minimal extension $T$ of the theory of divisible ordered abelian groups in the language $\mathscr{L}$. If $M \prec N$ are models of $T$ and $A \subseteq N$, then we write $M\langle A\rangle$ for the definable closure $\operatorname{dcl}(M \cup A)$ of $M \cup A$ in $N$ (which itself is an elementary substructure of $N$ ).

If $f: M^{n} \longrightarrow M$ is a definable map of a model $M$ of $T$, then $f$ extends to a map $S_{n}(M) \longrightarrow S_{1}(M)$ from the $n$-types $S_{n}(M)$ to the 1 -types of $M$, which we denote by $f$ again. By o-minimality, the set $S_{1}(M)$ of 1-types of $M$ can be viewed as the disjoint union of $M$ with the cuts of $M$. Moreover the definable non-isolated 1-types are precisely the principal cuts.

If $p$ and $q$ are cuts of $M$, then we say $p$ is equivalent to $q$ and write $p \sim q$, if there is a definable map $f: M \longrightarrow M$ with $f(p)=q$. By [Ma], Lemma 3.1, the relation $\sim$ is an equivalence relation between the cuts of $M$. Observe that $p \sim q$ if and only if $q$ is realized in $M\langle\alpha\rangle$ for some (or any) realization of $p$.

The easiest class of cuts after the principle cuts are described next.

(2.2) Definition. A cut $p$ of a model $M$ of $T$ is called dense if $p$ is not principal and $M$ is dense in $M\langle\alpha\rangle$, for some realization $\alpha$ of $p$.

In [Tr1], (3.6) other descriptions of density are given, e.g.: a non-principal cut is dense if and only is it has a principal heir. Important for us is: $p$ is dense if and only if $p$ is not principal and $G(p)=\{0\}$. In particular, dense cuts have signature 0 . In [We1], dense cuts are called "non-valuational".

(2.3) TheOREM. ([Tr1], (3.8))

Let $A \prec M, N$ be models of $T$ and let $p, q$ be dense cuts of $M, N$, respectively. Then $\left(M, p^{L}\right) \equiv_{A}\left(N, q^{L}\right)$ if and only if $p \nmid A=q \uparrow A$. Hence if $T$ has quantifier elimination and a universal system of axioms, then the $\mathscr{L}(\mathscr{D})$-theory $T^{\text {dense }}$ which expands $T$ and says that $\mathscr{D}$ is a set $p^{L}$ of a dense cut $p$, has quantifier elimination. Observe that density of $p$ can be expressed by a single sentence: we say that $G(p)=\{0\}$.

Recall from [Poi], 12.b, that a coheir of a type $p$ over an arbitrary first order structure $M$ is a type extending $p$ on some elementary extension of $M$, which is finitely realizable in $M$. From the identification of nonisolated 1-types and cuts of an o-minimal structure $M$, it follows easily that $q$ is a coheir of the nonprincipal cut $p$ of $M$ on $N \succ M$ if and only if $q$ is the least or the largest extension of $p$ on $N$.

The following proposition rephrases the signature of a cut $p$ of $M$ in terms of the behavior of the invariance groups of the least and the largest extension of $p$ on elementary extensions of $M$. 
(2.4) Proposition. Let $p$ be a cut of $M \models T$.

(i) $\operatorname{sign} p \geq 0$ if and only if for all $N \succ M$, if $q$ is the largest extension of $p$ on $N$, then the cut $\hat{q}$ is the largest extension of $\hat{p}$ on $N$.

(ii) $\operatorname{sign} p \leq 0$ if and only if for all $N \succ M$, if $q$ is the least extension of $p$ on $N$, then the cut $\hat{q}$ is the largest extension of $\hat{p}$ on $N$.

(iii) If $q$ is the least or the largest extension of $p$ on some $N \succ M$, then $\hat{q}$ is the least or the largest extension of $\hat{p}$ on $N$.

(iv) If $\operatorname{sign} p=0$ and $\alpha$ is a realization of $p$ in some $N \succ M$, then the map $2 \alpha-x$ swaps the least extension of $p$ on $N$ and the largest extension of $p$ on $N$. Moreover, if $H$ is the largest convex subgroup of $N$ with $H \cap M=G(p)$, then $\alpha-H^{+}, \alpha+H^{+}$are the least and the largest extension of $p$ on $N$.

(v) If $p$ is omitted in $N \succ M$ then $\operatorname{sign} q=\operatorname{sign} p$ for the unique extension $q$ of $p$ on $N$.

Proof. By [Tr1], (3.12). The moreover part in (iv) is shown in the proof of [Tr1], (3.12)(iv).

(2.5) Definition. Let $M, N$ be models of $T$ and let $A \prec M, N$ be a common elementary substructure. Let $p_{1}, \ldots, p_{n}$ be mutually distinct cuts of $M$ and let $q_{1}, \ldots, q_{n}$ be mutually distinct cuts of $N$. Let $\mathcal{D}_{1}, \ldots, \mathcal{D}_{n}$ be new unary predicates. We say the tuple $\left(q_{1}, \ldots, q_{n}\right)$ is an heir of $\left(p_{1}, \ldots, p_{n}\right)$ over $A$ if the following condition holds: if $\varphi\left(x_{1}, \ldots, x_{k}\right)$ is an $\mathscr{L}$ formula with parameters from $A, \psi\left(x_{1}, \ldots, x_{k}\right)$ is a quantifier free $\mathscr{L}\left(\mathcal{D}_{1}, \ldots, \mathcal{D}_{n}\right)$-formula with parameters from $A$ and if

$$
\left(N, q_{1}^{L}, \ldots, q_{n}^{L}\right) \models \exists \bar{x} \varphi(\bar{x}) \wedge \psi(\bar{x})
$$

then

$$
\left(M, p_{1}^{L}, \ldots, p_{n}^{L}\right) \models \exists \bar{x} \varphi(\bar{x}) \wedge \psi(\bar{x}) .
$$

If $A=M$ we say that $\left(q_{1}, \ldots, q_{n}\right)$ is an heir of $\left(p_{1}, \ldots, p_{n}\right)$. For example if $q$ is an $n$-type with projections $q_{1}, \ldots, q_{n}$ and $q$ is an heir of $q\lceil M$ in the sense of model theory (cf. [Poi], 11.a), then $\left(q_{1}, \ldots, q_{n}\right)$ is an heir of $\left(p_{1}, \ldots, p_{n}\right)$. The precise relation with the model theoretic notion of an heir is described in $[\operatorname{Tr} 1],(2.7)$.

Notice that already in the case $n=2$, if $q_{i}$ is an heir of $p_{i}(i=1,2)$, then $\left(q_{1}, q_{2}\right)$ is in general not an heir of $\left(p_{1}, p_{2}\right)$, even if $\left(p_{1}, p_{2}\right)$ and $\left(q_{1}, q_{2}\right)$ determine unique 2 -types. One reason is that formulas $\exists \bar{x} \varphi(\bar{x}) \wedge \psi(\bar{x})$ as in the definition above can express that $q_{1}$ is strictly larger than $\hat{q}_{2}$, i.e. there are $\alpha, \beta \in N$ with $0<\alpha<q_{1}$ and $\beta<q_{2}<\alpha \cdot \beta$ whereas in general, $p_{1}$ might be equal to $\hat{p}_{2}$, i.e. there are no $a, b \in M$ with $0<a<p_{1}$ and $b<p_{2}<a \cdot b$. This is worked out in the example (4.3), where we work multiplicatively and $p_{2}, q_{2}$ are even upper edges of convex subgroups.

(2.6) Remark.([Tr1], (2.8)) Let $p_{1}, \ldots, p_{n}$ be pairwise distinct cuts of $M=T$ and let $q_{1}, \ldots, q_{n}$ be extensions of $p_{1} \uparrow A, \ldots, p_{n} \uparrow A$ on $N \succ M$, respectively, with $q_{i} \notin\left\{ \pm \infty_{N}\right\}$. Then $\left(q_{1}, \ldots, q_{n}\right)$ is an heir of $\left(p_{1}, \ldots, p_{n}\right)$ over $A$ if and only if the following condition holds:

if $\varphi\left(u_{1}, \ldots, u_{n}, v_{1}, \ldots, v_{n}\right)$ is an $\mathscr{L}$-formula with parameters from $A$ and $\bar{\alpha}, \bar{\beta} \in N^{n}$ such that $\alpha_{1}<q_{1}<\beta_{1}, \ldots, \alpha_{n}<q_{n}<\beta_{n}$ and $N \models \varphi(\bar{\alpha}, \bar{\beta})$, then there are $\bar{a}, \bar{b} \in M^{n}$ such that $a_{1}<p_{1}<b_{1}, \ldots, a_{n}<p_{n}<b_{n}$ and $M=\varphi(\bar{a}, \bar{b})$. 
(2.7) LEMmA.([Tr1], (2.10)) If $p$ is a nonprincipal cut of $M \models T$, then both coheirs of $p$ on $N \succ M$ are heirs of $p$. If in addition $\operatorname{dim} N / M=1$, then these are the only heirs of $p$ on $N$.

To see what happens in $\operatorname{dim} N / M \geq 2$, take $M \prec M_{1} \prec N$, let $p$ be a cut of $M$, let $p_{1}$ be the largest extension of $p$ on $M_{1}$ and let $q$ be the least extension of $p_{1}$ on $N$. If $M_{1}$ contains a realization of $p$, then $q$ is not the least extension of $p$ on $N$. If $N$ contains a realization of $p_{1}$, then $q$ is not the largest extension of $p$ on $N$. Clearly both assumptions may be true already when $\operatorname{dim} N / M=2$. By (2.7), $p_{1}$ is an heir of $p$ and $q$ is an heir of $p_{1}$ (assuming that $p$ and $p_{1}$ are not principal, which holds true for example if $p$ is the upper edge of a proper convex subgroup of $M$ ). Hence also $q$ is an heir of $p$, whereas $q$ is not a coheir of $p$.

(2.8) REMARK. Recall that until now we are working with an o-minimal extension of ordered abelian groups. Therefore the statements above are also applicable multiplicatively when $T$ is an o-minimal expansion of a real closed field. For example (2.4)(iv) then says: If $\operatorname{sign}^{*} p=0, \alpha \in N \succ M$ is a realization of $p$ and $H$ is the largest convex subgroup of $\left(N^{>0}, \cdot, \leq\right)$ with $H \cap M=G^{*}(p)$, then $\alpha \cdot H^{+}, \alpha \cdot H^{-}$are the least and the largest extension of $p$ on $N$. A more formal explanation can be found in [Tr1], (5.3).

For the rest of this paper we work with an o-minimal, polynomially bounded theory $T$ in the language $\mathscr{L}$, which has an archimedean prime model.

In the remaining part of this section we record facts which are used later on. Some of them are minor alterations of the original statements, in these cases we include proofs explaining the amendments. Notice that the current section can be used, but is not intended to serve as, an introduction to the model theory of cuts of polynomially bounded structures so far. The main purpose is to provide precise statements ready for citation exactly as they are needed in some of the technical parts later.

Recall: "polynomially bounded" means that for each 0-definable map $f$ there is some $n \in \mathbb{N}$ with $f(x) \leq x^{n}$ for sufficiently large $x$. For example real closed fields are polynomially bounded.

Moreover, the field of exponents of $T$ is the subfield of $\mathbb{R}$ consisting of all $\lambda \in \mathbb{R}$ such that the function $x \mapsto x^{\lambda}$, defined on the positive elements of the prime model of $T$, is definable in $T$. The elements of the field of exponents of $T$ are called exponents. For example the field of exponents of the theory of real closed fields is $\mathbb{Q}$.

We shall use facts about convex valuation rings of models of $T$ from [vdD-Lew], which we collect now. Note: since $T$ is assumed to be polynomially bounded with archimedean prime model, every convex subring of a model of $T$ is " $T$-convex". We don't use this notion explicitly and specialize results of [vdD-Lew], section 2 to our situation:

(2.9) FACTs. Let $M$ be a model of $T$ and let $V$ be a convex subring of $M$ with maximal ideal $\mathfrak{m}$.

1. For every continuous, 0-definable map $f: M^{n} \longrightarrow M$ we have $f\left(V^{n}\right) \subseteq V$.

2. A structure $M_{0} \prec M$ with $M_{0} \subseteq V$ is maximal such if and only if the composition $M_{0} \longrightarrow V \longrightarrow V / \mathfrak{m}$ is an isomorphism if and only if $M_{0}$ is tame in $M$, i.e. every $M_{0}$-bounded element of $M$ is infinitely close (in the sense of $M_{0}$ ) to an element of $M_{0}$. If this is the case, we install the $\mathscr{L}$-structure of $M_{0}$ via the isomorphism $M_{0} \longrightarrow V / \mathfrak{m}$ on $V / \mathfrak{m}$ and $V / \mathfrak{m}$ becomes a model of $T$ which does not depend on the choice of $M_{0}$.

3. Let $M \prec N$ and let $W \subseteq N$ be a convex subring, with maximal ideal $\mathfrak{n}$, lying over $V$ (i.e. $W \cap M=V$ ). Then the canonical morphism $V / \mathfrak{m} \longrightarrow W / \mathfrak{n}$ is an elementary map of models of $T$. Moreover, the dimension of $W / \mathfrak{n}$ over $V / \mathfrak{m}$ does not exceed $\operatorname{dim} N / M$. 
4. If $M_{0} \prec M$ is contained in $V$ and $\alpha \in V, \alpha>M_{0}$, then $M_{0}\langle\alpha\rangle \subseteq V$.

Let $\mathscr{L}_{\text {convex }}$ be the language $\mathscr{L}$, expanded by a new unary predicate $\Theta$ (for the convex valuation ring) and let $T_{\text {convex }}$ be the $\mathscr{L}_{\text {convex }}$-theory of pairs $(M, V)$, where $M \models T$ and $V$ is a proper convex valuation ring of $M$.

(2.10) TheOREM. ([vdD-Lew], (3.10), (3.13) and (3.14))

$T_{\text {convex }}$ is a complete $\mathscr{L}_{\text {convex }}$-theory. $T_{\text {convex }}$ is weakly o-minimal (i.e. each $M$-definable subset of a model $(M, V)$ of $T_{\text {convex }}$ is a finite union of convex subsets of $\left.M\right)$. If $T$ has quantifier elimination and a universal system of axioms, then $T_{\text {convex }}$ has quantifier elimination.

In fact, weak o-minimality is also valid in every expansion $(M, \mathscr{C})$ of $M \models T$ by an arbitrary set $\mathscr{C}$ of convex subsets of $M$. This follows from a result of Baisalov and Poizat (cf. [Ba-Po]), which was later generalized by Shelah (cf. [She2]):

(2.11) Theorem. Let $M$ be an o-minimal structure and let $\mathscr{M}$ be the expansion of $M$ by all sets $Z \cap M^{n}$, where $Z$ runs through the definable subsets of elementary extensions of $M$. Then $T h(\mathscr{M})$ is weakly o-minimal with quantifier elimination.

We shall frequently work in the language $\mathscr{L}^{d f}$ with the theory $T^{d f}$ (cf. [vdD-Lew], (2.3) and (2.4)), which is an extension by definitions of $T$, where we have a function symbol for each 0-definable function $M^{n} \longrightarrow M$. Since $T$ has definable Skolem functions the theory $T^{d f}$ has quantifier elimination and a universal system of axioms.

The following theorem explicitly lists all cuts, definable in $(M, V)$, for a convex subring $V$ of $M$. This is one starting point in the analysis of cuts in [Tr1].

(2.12) THEOREM. ([Tr2], (2.12))

Let $M$ be a model of $T$, let $V$ be a convex valuation ring of $M$ with maximal ideal $\mathfrak{m}$ and let $A$ be a subset of $M$. If $p$ is a nonprincipal cut of $M$, such that $p^{L}$ is A-definable in $(M, V)$, then there are $a, b \in \operatorname{dcl}(A), b \neq 0$ such that $p=a+b \cdot V^{+}$or $p=a+b \cdot \mathfrak{m}^{+}$. Moreover, $V \subseteq G(p)$ or $G(p) \subseteq \mathfrak{m}$.

A key property of the model theory of cuts of polynomially bounded structure is the next

(2.13) TheOREM. Let $p$ be a cut of a model $M$ of $T$.

(i) $\operatorname{sign} p=0 \Leftrightarrow p \nsim G^{+}$for every convex subgroup of $(M,+, \leq)$.

(ii) $\operatorname{sign}^{*} \hat{p}=0 \Leftrightarrow \hat{p} \nsim V^{+}$for every convex valuation ring $V$ of $M$.

Proof. (i) holds by [Tr2], (4.3).

(ii) follows from (2.12): clearly we have $\Rightarrow$. In order to see $\Leftarrow$ assume $\hat{p} \sim V^{+}$. Then $\hat{p}^{L}$ is definable in $(M, V)$, thus by $(2.12), \hat{p}=a+b \cdot V^{+}$or $\hat{p}=a+b \cdot \mathfrak{m}^{+}$for some $a, b \in M$. Since $\hat{p}$ is the upper edge of a convex subgroup we obtain $\hat{p}=b \cdot V^{+}$or $\hat{p}=b \cdot \mathfrak{m}^{+}$, which means $\operatorname{sign}^{*} \hat{p}=1$ or $\operatorname{sign}^{*} \hat{p}=-1$.

Theorem (2.13) says that a given cut $p$ can be mapped onto $\hat{p}$ via a linear map $a \pm x$ if there is a definable function $f$ and a group $G$ at all with $f(p)=G^{+}$(and similarly for $\hat{p}$ ). Note hat $f(p)=G^{+}$does not imply $G=G(p)$ since for example $\left(G^{+}\right)^{2} \neq G^{+}$unless $G$ is a valuation ring or a maximal ideal of a valuation ring. In particular, equivalence of cuts does not preserve the invariance groups. On the other hand we shall see in (5.2), that equivalence of cuts does preserve the invariance rings. 
(2.14) Corollary. Let $p$ be a cut of a model $M$ of $T$ and let $\alpha$ be a realization of $p$ from some elementary extension of $M$.

(i) $\operatorname{sign} p=0$ if and only if for every realization $\beta \in M\langle\alpha\rangle$ of $p$ there is some $m \in G(p)$ with $\beta-\alpha \leq m$.

(ii) Here we assume $p=\hat{p}$. Then $\operatorname{sign}^{*} p=0$ if and only if for every realization $\beta \in M\langle\alpha\rangle$ of $p$ there is some $m \in V(p)$ with $\frac{\beta}{\alpha} \leq m$.

Proof. (i). $\Rightarrow$. Take a realization $\beta \in M\langle\alpha\rangle$ of $p$. Since $\alpha$ and $\beta$ realize $p$, there is no element $m \in M, m>G(p)$ with $m<\beta-\alpha$. Therefore $q \leq \hat{p}$ for the cut $q$ of $M$ realized by $\beta-\alpha$. As $\operatorname{sign} p=0$ we have $p \nsim \hat{p}$ by (2.13)(i), hence $\hat{p}$ is omitted in $M\langle\alpha\rangle$. Thus $q<\hat{p}$, which gives the claim.

$\Leftarrow$. Suppose $\operatorname{sign} p=1$, hence $p=a+\hat{p}$ for some $a \in M$ and $\alpha-a \models \hat{p}$. Since $\hat{p}$ is the upper edge of a convex subgroup of $(M,+, \leq)$ and $(M,+, \leq)$ is divisible, also $2(\alpha-a) \models \hat{p}$. Hence $\beta:=a+2(\alpha-a)=2 \alpha-a$ realizes $p$. Since $\beta-\alpha=\alpha-a$ is a realization of $\hat{p}$ there is no $m<\hat{p}$ with $\beta-\alpha \leq m$.

Suppose $\operatorname{sign} p=-1$, hence $p=a-\hat{p}$ for some $a \in M$ and $a-\alpha=\hat{p}$. Since $\hat{p}$ is the upper edge of a convex subgroup of $(M,+, \leq)$ and $(M,+, \leq)$ is divisible, also $\frac{a-\alpha}{2} \models \hat{p}$. Hence $\beta:=a-\frac{a-\alpha}{2}=\frac{a+\alpha}{2}$ realizes $p$. Since $\beta-\alpha=\frac{a+\alpha}{2}-\alpha=\frac{a-\alpha}{2}$ is a realization of $\hat{p}$ there is no $m<\hat{p}$ with $\beta-\alpha \leq m$.

Item (ii) holds by the same proof, written multiplicatively using (2.13)(ii) and the fact that $\left(M^{>0}, \cdot,<\right)$ is divisible. Notice that $V(p)^{+}=G^{*}(p)^{+}$, since $p=\hat{p}$.

Without the assumption $\beta \in M\langle\alpha\rangle$ it is not true that $\operatorname{sign} p=0$ implies the existence of $m \in G(p)$ with $\beta-\alpha<m$. For example if $p$ is the cut of $\pi$ over the real algebraic numbers, $\alpha=\pi$ and $\beta$ realizes the largest extension of $p$ on $\mathbb{R}_{\mathrm{alg}}\langle\pi\rangle$.

The next proposition gives a method to produce cuts $p$ with prescribed values of sign $p$ and $\operatorname{sign}^{*} \hat{p}$. This is mainly used when looking for (counter-)examples.

(2.15) Proposition. ([Tr1], (6.5) and [Tr1], (6.6))

Let $M$ be a model of $T$ and let $G$ be a convex subgroup of $(M,+, \leq)$. Let $V$ be a convex valuation ring of $M$ with $V \subseteq V(G)$. Let $\mathscr{C}$ be a set of cuts of $M$ with $G \subseteq G(p)$ for all $p \in \mathscr{C}$. Let $\varepsilon, \delta \in\{-1,0,1\}$.

Then there are an elementary extension $N$ of $M$ with $\operatorname{dim} N / M=\aleph_{0}+|\mathscr{C}|$ and a convex subgroup $H$ of $(N,+, \leq)$, such that $H \cap M=G$, sign* $H^{+}=\varepsilon, V(H)^{+}$is an heir of $V^{+}$and such that for each $p \in \mathscr{C}$ there is a cut $q$ of $N$ extending $p$ with $\operatorname{sign} q=\delta$ and $G(q)=H$.

Moreover, if $\varepsilon=0$, then we can choose $H$ so that in addition, $V(H)$ is the convex hull of $V$ in $N$.

In particular, for every cut $p$ of a model $M$ of $T$ and all $\varepsilon, \delta \in\{-1,0,1\}$ there is a cut $q$ on some elementary extension of $M$ such that

(a) $q$ extends $p, \hat{q}$ extends $\hat{p}$ and $V(q)^{+}$extends $V(p)^{+}$.

(b) $\operatorname{sign} q=\delta$ and $\operatorname{sign}^{*} \hat{q}=\varepsilon$.

Here a complete description of heirs of cuts in terms of their invariance groups and invariance rings as proved in [Tr1]: 
(2.16) THEOREM. Let $p$ be a cut of a model $M$ of $T$ and let $q$ be a cut extending $p$ on some $N \succ M$.

(i) If $p=a \pm \hat{p}$ for some $a \in M$, then $q$ is an heir of $p$ if and only if $q=a \pm \hat{q}$ and $\hat{q}$ is an heir of $\hat{p}$.

(ii) If $\operatorname{sign} p=0$, then $q$ is an heir of $p$ if and only if $\hat{q}$ extends $\hat{p}$.

(iii) If $p=\hat{p}$ and $\operatorname{sign}^{*} p=0$, then $q$ is an heir of $p$ if and only if $q=\hat{q}$ and $V(q)$ lies over $V(p)$. If this is the case, then also $\left(V(q)^{+}, q\right)$ is an heir of $\left(V(p)^{+}, p\right)$.

(iv) If $p$ is a dense cut, i.e. $\operatorname{sign} p=0$ and $G(p)=\{0\}$, then $\left(V(q)^{+}, \hat{q}, q\right)$ is an heir of $\left(V(p)^{+}, \hat{p}, p\right)$ if and only if $G(q)=\{0\}$.

(v) If $\operatorname{sign} p=0, G(p) \neq\{0\}$ and $\operatorname{sign}^{*} \hat{p}=0$, then $\left(V(q)^{+}, \hat{q}, q\right)$ is an heir of $\left(V(p)^{+}, \hat{p}, p\right)$ if and only if $\hat{q}$ extends $\hat{p}$ and $V(q)$ lies over $V(p)$.

(vi) If $\operatorname{sign} p=0, G(p) \neq\{0\}$ and $\operatorname{sign}^{*} \hat{p} \neq 0$, then $\left(V(q)^{+}, \hat{q}, q\right)$ is an heir of $\left(V(p)^{+}, \hat{p}, p\right)$ if and only if $\hat{q}$ extends $\hat{p}, V(q)$ lies over $V(p)$ and if there is some $a \in M$ such that $\hat{q}$ is an edge of $a \cdot V(q)^{*,>0}$.

Proof. (i) is easy and can be found in [Tr1], (3.14)(ii). For the remaining statements recall that all convex subrings of all models of $T$ are $T$-convex. We then may apply (2.13) together with results from [Tr1]:

(ii) holds by [Tr1], (3.14)(iii). (iii) holds by [Tr1], (3.14)(iii), applied to the o-minimal structure induced by $M$ on the multiplicative group of positive elements of $M$ (cf. [Tr1], (5.3)). (iv) holds by [Tr1], (3.6). (v) and (vi) hold by [Tr1], (7.3).

Theorem (2.16) naturally leads to model completeness results:

(2.17) Theorem. ([Tr2], (2.16))

Let $T$ be model complete and let $\Theta, \mathscr{G}, \mathscr{Z}, \mathscr{Z}^{*}$ and $\mathscr{D}$ be new unary predicates. Let $\varepsilon, \delta \in$ $\{-1,0,1\}$ and let $\mathscr{L}^{*}$ be the language $\mathscr{L}\left(\Theta, \mathscr{G}, \mathscr{Z}, \mathscr{Z}^{*}, \mathscr{D}\right)$. Let $T_{\delta}^{\varepsilon}$ be the $\mathscr{L}^{*}$-theory which extends $T$ and which says the following things about a model $\left(M, V, G, Z, Z^{*}, D\right)$ :

(a) $D=p^{L}$ for some cut $p$ of $M$, $p$ neither dense nor principal with $\operatorname{sign} p=\delta$.

(b) $V=V(p)$.

(c) $G=G(p)$ and $\operatorname{sign}^{*} G^{+}=\varepsilon$.

(d) $Z=\left\{a \in M \mid a+G^{+}=p\right.$ or $\left.a-G^{+}=p\right\}$ and

$Z^{*}=\left\{a \in M^{>0} \mid a \cdot V=G\right.$ or $\left.a \cdot \mathfrak{m}=G\right\}$, where $\mathfrak{m}$ is the maximal ideal of $V$.

Then $T_{\delta}^{\varepsilon}$ is model complete (and consistent).

(2.18) Corollary. ([Tr2], (2.17))

Let $T$ be model complete in the language $\mathscr{L}$. Let $\Theta, \mathscr{G}, \mathscr{Z}, \mathscr{Z} *$ and $\mathscr{D}$ be new unary predicates.

(i) The $\mathscr{L}(\Theta, \mathscr{G})$-theory $T_{\text {group }}^{0}$ which extends $T$ and which says that $\mathscr{G}$ is a convex subgroup, such that the upper edge of $\mathscr{G}$ has multiplicative signature 0 and invariance ring $\theta$, is model complete.

(ii) The $\mathscr{L}(\Theta, \mathscr{G}, \mathscr{D})$-theory $T_{\text {cut }}^{0,0}$ which extends $T_{\text {group }}^{0}$ and which says that $\mathscr{D}$ is the set $p^{L}$ of a cut $p$ of signature 0 and invariance group $\mathscr{G}$, is model complete.

We conclude this section with a pocket guide containing the most important properties of cuts, frequently used in the text afterwards. $p$ always denotes a cut of a polynomially bounded structure $M, M\langle p\rangle$ is the definable closure of $M$ together with a realization of $p$, $\hat{p}=G(p)^{+}$and $V(p)=V(G(p))$. 
$p$ principal : Equivalently: $\bullet p= \pm a$ or $p= \pm \infty \bullet p$ is definable $\bullet p$ has a unique heir on all $N \succ M \bullet M$ is Dedekind complete in $M\langle p\rangle \bullet M$ is not cofinal in $M\langle p\rangle$. Properties: • $G\left(a^{ \pm}\right)=\{0\}, G( \pm \infty)=M \bullet V(p)=M$.

$p$ dense : $\quad$ Equivalently: $\bullet p$ not definable and $G(p)=\{0\} \bullet p$ not definable and $V(p)=$ $M \bullet p$ not definable and $p$ has a principal heir $\bullet M$ is dense in $M\langle p\rangle$. Properties: $p \nsim \hat{p}$.

$\operatorname{sign} p= \pm 1:$ Equivalently: $\bullet p=a \pm \hat{p} \bullet p \sim \hat{p} \bullet p \sim G^{+}$for some group $G$.

Properties: $\bullet p=a \pm G^{+} \Rightarrow G=G(p) \bullet q$ heir of $p$ iff $q=a \pm \hat{q}$ and $\hat{q}$ heir of $\hat{p}$.

$\operatorname{sign} p=0: \quad$ Equivalently: $\bullet p \not \hat{p} \bullet p \not G^{+}$for every group $G$.

Properties: • not definable $\bullet q$ heir of $p$ iff $\hat{p} \subseteq \hat{q}$.

$p \sim q: \quad$ Equivalently: $\bullet f(p)=q$ for some definable $f: M \longrightarrow M$

- $q \sim p \bullet q$ realized in $M\langle p\rangle$.

$p \nsim q$ : $\quad$ Equivalently: $\bullet q \not p \bullet q$ has a unique extension on $M\langle p\rangle$.

$\underline{\text { sign }^{*} \hat{p}= \pm 1}:$ Equivalently: $G(p)=a \cdot V(p)$ or $G(p)=a \cdot \mathfrak{m}(p)$.

$\operatorname{sign}^{*} \hat{p}=0: \quad$ Equivalently: $\hat{p} \nsim V(p)^{+}$.

Properties: $q$ heir of $\hat{p}$ iff $q=\hat{q}$ and $V(p) \subseteq V(q)$.

\section{BOX TYPES}

We first recall from [Tr3], section 1, some properties of a dimension in o-minimal structures which is a proper coarsening of the ordinary dimension obtained from the definable closure.

(3.1) Definition. Let $M$ be o-minimal and let $p$ be an $n$-type of $M$. We say that $p$ is a box type if $p$ is uniquely determined by those formulas from $p$ which define the open boxes $\prod_{i=1}^{n}\left(a_{i}, b_{i}\right), a_{i}, b_{i} \in M \cup\{ \pm \infty\}$.

(3.2) Proposition. Let $M$ be o-minimal (not necessarily polynomially bounded). Let $p \in$ $S_{n}(M)$ and suppose that the projections $p_{1}, \ldots, p_{n}$ of $p$ onto the coordinate axes are cuts of M. The following are equivalent:

(i) For each $k \in\{1, \ldots, n\}$ and all realizations $\alpha_{1}, \ldots, \alpha_{k-1}$ of $p_{1}, \ldots, p_{k-1}$ respectively, $p_{k}$ has a unique extension on $M\left\langle\alpha_{1}, \ldots, \alpha_{k-1}\right\rangle$.

(ii) $p$ is a box type.

(iii) There is a unique $n$-type of $M$ with projections $p_{1}, \ldots, p_{n}$, namely $p$.

ProOF. Item (i) says that for some (hence for each) realization $\bar{\alpha}$ of $p$, the realization rank of $\bar{\alpha}$ over $M$, as defined in [Tr3], section 1 , is equal to $n$. Now the proposition is [Tr3], (1.15).

(3.3) REMARKS.

(i) If $p, q$ are cuts of $M$, then $p \nsim q$ if and only if $q$ is omitted in $M\langle\alpha\rangle$ for every realization $\alpha$ of $p$. Hence by (3.2) $p, q$ of $M$ are not equivalent if and only if $(p, q)$ is a box type.

(ii) It follows that the property of $p_{1}, \ldots, p_{k}$ stated in (3.2)(i) also holds for any permutation of $p_{1}, \ldots, p_{k}$, since this is certainly true for item (iii) of (3.2). 
(iii)In view of (3.2)(iii), we shall say " $\left(p_{1}, \ldots, p_{n}\right)$ is a box type" whenever $p_{1}, \ldots, p_{n}$ are cuts of $M$, which determine a box type $p \in S_{n}(M)$.

If this is the case and $f: M^{n} \longrightarrow M$ is $M$-definable, then we write $f\left(p_{1}, \ldots, p_{n}\right)$ for the 1-type $f(p)$.

(3.4) Corollary. Let $p_{1}, \ldots, p_{n}, q_{1}, \ldots, q_{k}$ be cuts of $M=T$ such that $\left(q_{1}, \ldots, q_{k}, p_{i}\right)$ is a box type $(1 \leq i \leq n)$ and let $\alpha_{1}, \ldots, \alpha_{k}$ be realizations of $q_{1}, \ldots, q_{k}$, respectively. Let $p_{i}^{\prime}$ be the unique extension of $p_{i}$ on $M\left\langle\alpha_{1}, \ldots, \alpha_{k}\right\rangle(1 \leq i \leq n)$. Then $\left(p_{1}^{\prime}, \ldots, p_{n}^{\prime}\right)$ is a box type if and only if $\left(q_{1}, \ldots, q_{k}, p_{1}, \ldots, p_{n}\right)$ is a box type.

Proof. Easily from $(3.2)(\mathrm{i}) \Leftrightarrow($ ii).

(3.5) Proposition. Let $M$ be a model of $T$ and let $p, q$ be cuts of $M$ such that $p$ is not principal or $q$ is not dense. Let $M^{\prime} \succ M$ such that $p$ is omitted in $M^{\prime}$ and $q$ is realized in $M^{\prime}$. Let $p^{\prime}$ be the unique extension of $p$ on $M^{\prime}$ and let $f: M^{\prime} \longrightarrow M^{\prime}$ be $M^{\prime}$-definable.

If $f\left(p^{\prime}\right)$ extends $q$, then there is some $a \in M$ such that $f(a)$ realizes $q$.

PROOF. By the monotonicity theorem for o-minimal structures we may assume that $f$ is constant or strictly monotonic in some $M^{\prime}$-definable open interval $I$ containing $p^{\prime}$. Since $p$ is omitted in $M^{\prime}$ we may assume that $I$ has endpoints in $M \cup\{ \pm \infty\}$. If $f$ is constant in $I$, say $=\beta$, then $f(a)=\beta \models q$ for every $a \in I$ and we are done. Hence we may assume that $f$ is strictly monotonic in $I$ and $f\left(p^{\prime}\right)$ is a cut of $M^{\prime}$.

Case 1. $p$ is principal, say $p=+\infty_{M}$.

Then $p^{\prime}=+\infty_{M^{\prime}}$ and $f\left(p^{\prime}\right)$ can not be $\pm \infty_{M^{\prime}}$ : otherwise, as $f\left(p^{\prime}\right)$ extends $q, q= \pm \infty_{M}$ which contradicts our assumptions: $p$ is omitted and $q$ is realized in $M^{\prime}$.

Thus by o-minimality the $\operatorname{limit} \lim _{t \rightarrow \infty} f(t)$ exists in $M^{\prime}$, call it $\beta$. By assumption, $q$ is not dense, in other words there is some $c \in M, c>0$ such that $c+q=q$. Since $f\left(p^{\prime}\right)$ extends $q$, it follows that every element in $(\beta-c, \beta+c) \subseteq M^{\prime}$ is a realization of $q$. Since $\lim _{t \rightarrow \infty} f(t)=\beta, f(x)$ is a realization of $q$ for all sufficiently large elements in $M^{\prime}$. Since $M$ is cofinal in $M^{\prime}$, there is some $a \in M$ with $f(a) \models q$.

Case 2. $p$ is not principal.

Then $C:=\left\{\alpha \in I \mid \alpha<p^{\prime}\right\}$ and $D:=\left\{\alpha \in I \mid p^{\prime}<\alpha\right\}$ both are convex sets with infinitely many points. Take a realization $\beta \in M^{\prime}$ of $q$, say $\beta<f\left(p^{\prime}\right)$. Since $f$ is strictly monotonic in $I=C \cup D$ and $C, D$ are infinite, $f\left(p^{\prime}\right)$ is the unique cut of $M^{\prime}$ between $f(C)$ and $f(D)$. Hence there must be some $\alpha \in I$ with $\beta \leq f(\alpha)<f\left(p^{\prime}\right)$. Since $p$ is omitted in $M^{\prime}$, there is some $a \in M$ between $\alpha$ and $p^{\prime}$. Then $f(a)$ lies between $f(\alpha)$ and $f\left(p^{\prime}\right)$, thus $\beta \leq f(a)<f\left(p^{\prime}\right)$. Since $\beta$ realizes $q$ and $f\left(p^{\prime}\right)$ extends $q, f(a)$ also realizes $q$.

Observe that the assumption " $p$ is not principal or $q$ is not dense" in (3.5) is necessary: if $p=+\infty_{M}$ and $q$ is a dense cut of $M$, realized by $\beta$, take $M^{\prime}=M\langle\beta\rangle$ and $f(x):=\beta+\frac{1}{x}$; then $p^{\prime}=+\infty_{M^{\prime}}$ and $f\left(p^{\prime}\right)=\beta^{+}$extends $q$, but as $G(q)=\{0\}$ there is no $a \in M$ such that $f(a) \models q$.

(3.6) Corollary. Let $\left(p_{1}, \ldots, p_{n}, q_{1}, \ldots, q_{k}\right)$ be a box type of $M \models T$ and let $f: M^{n} \times M^{k} \longrightarrow$ $M, g: M^{n} \longrightarrow M$ be $M$-definable such that $f\left(p_{1}, \ldots, p_{n}, q_{1}, \ldots, q_{k}\right)=g\left(p_{1}, \ldots, p_{n}\right)$. Suppose none of the $q_{i}$ is principal or $g\left(p_{1}, \ldots, p_{n}\right)$ is not dense. Then there are $a_{1}, \ldots, a_{k} \in M$ with

$$
f\left(p_{1}, \ldots, p_{n}, a_{1}, \ldots, a_{k}\right)=g\left(p_{1}, \ldots, p_{n}\right) .
$$

Proof. The corollary obviously follows inductively from the case $k=1$. Let $\bar{\alpha}$ be a realization of $\left(p_{1}, \ldots, p_{n}\right)$ and let $\beta$ be a realization of $q_{1}$. We apply (3.5) with $M^{\prime}=M\langle\bar{\alpha}\rangle$, 
$p:=q_{1}, q:=f\left(p_{1}, \ldots, p_{n}, q_{1}\right)$ and the $M^{\prime}$-definable map $f(\bar{\alpha}, x)$. Since $\left(p_{1}, \ldots, p_{n}, q_{1}\right)$ is a box type, $p$ is omitted in $M^{\prime}$. Moreover, $p$ is not principal or $q$ is not dense by assumption. Since $f\left(p_{1}, \ldots, p_{n}, q_{1}\right)=g\left(p_{1}, \ldots, p_{n}\right), q$ is realized in $M^{\prime}$ by $g(\bar{\alpha})$. Finally, $f\left(\bar{\alpha}, p^{\prime}\right)$ extends $q$ by definition of the data, where $p^{\prime}$ denotes the unique extension of $p$ on $M^{\prime}$.

Thus by (3.5), there is some $a \in M$ such that $f(\bar{\alpha}, a)$ realizes $q=f\left(p_{1}, \ldots, p_{n}, q_{1}\right)$, as desired.

\section{The BOX TYPE ASSOCIATED TO A CUT}

(4.1) Definition. For a cut $p$ of a real closed field $M$ we define the $p$-box to be

$$
\operatorname{box}(p):= \begin{cases}\left(p, V(p)^{+}\right) & \text {if } \operatorname{sign} p \neq 0 \text { and } \operatorname{sign}^{*} \hat{p}=0 \\ (p, \hat{p}) & \text { if } \operatorname{sign} p=0 \text { and } \operatorname{sign}^{*} \hat{p} \neq 0 \\ \left(p, \hat{p}, V(p)^{+}\right) & \text {if } \operatorname{sign} p=0 \text { and } \operatorname{sign}^{*} \hat{p}=0 \\ p & \text { otherwise. }\end{cases}
$$

Observe that $\operatorname{box}(p)=p$ if $p$ is principal and $\operatorname{box}(p)=\left(p, 0^{+}\right)$if $p$ is dense.

Recall that we are working with a polynomially bounded theory $T$ which has an archimedean prime model.

(4.2) Proposition. If $p$ is a cut of $M \models T$ then $\operatorname{box}(p)$ is a box type.

PROOF. If $\operatorname{sign} p \neq 0$ or $\operatorname{sign}^{*} \hat{p} \neq 0$, then we know this from (2.13) (recall that two cuts form a box type if and only if they are not equivalent). Hence we may assume that $\operatorname{sign} p=0$ and $\operatorname{sign}^{*} \hat{p}=0$, thus by (2.13) we have $p \not \hat{p}$ and $\hat{p} \not V(p)^{+}$.

Let $\gamma$ be a realization of $\hat{p}$. Since $p \not \hat{p}, p$ has a unique extension $r$ on $M\langle\gamma\rangle$ and by (2.4)(v), this extension has signature 0 again. Since $\hat{p} \nsim V(p)^{+}, V(p)^{+}$has a unique extension on $M\langle\gamma\rangle$, namely $W^{+}$, where $W$ is the convex hull of $V(p)$ in $M\langle\gamma\rangle$. By (2.4)(i), we know that $\hat{r}$ is the largest extension of $\hat{p}$ on $M\langle\gamma\rangle$. By (2.4)(i), applied multiplicatively to $\hat{p}$ and $\hat{r}$, it follows $W=V(r)$. From $\operatorname{sign} r=0$ and $(2.13)(\mathrm{i})$ we get that $\left(r, V(r)^{+}\right)$is a box type. By (3.4), $\left(p, \hat{p}, V(p)^{+}\right)$is a box type.

(4.3) ExAmple. We now give an example of a box type $\left(p_{1}, p_{2}\right) \in S_{2}(M)$ of a pure real closed field $M$, and a box type $\left(q_{1}, q_{2}\right) \in S_{2}(N)$ extending $\left(p_{1}, p_{2}\right)$ on $N \succ M$ such that $q_{i}$ is an heir of $p_{i}(i=1,2)$, but $\left(q_{1}, q_{2}\right)$ is not an heir of $\left(p_{1}, p_{2}\right)$ :

Take a real closed field $M$ and a convex subgroup $G$ of $(M,+, \leq)$ such that sign* $G^{+}=0$ (use (2.15) to find one or take $M=\mathbb{R}\left(\left(t^{\mathbb{Q}}\right)\right)$ and $G=\{x \in M \mid v(x)>-\pi\}$ ). By (4.2), $\left(V(G)^{+}, G^{+}\right)$is a box type and we may take $\left(p_{1}, p_{2}\right)=\left(V(G)^{+}, G^{+}\right)$. Let $\alpha$ be a realization of $G^{+}$and let $\omega$ be a realization of the upper edge of $V(G)$ in $M\langle\alpha\rangle$. Then $\alpha \cdot \omega$ still is a realization of $p_{2}$ : otherwise there is $m \in M$ with $\alpha<m<\alpha \cdot \omega$, hence $1<\frac{m}{\alpha}<\omega$; by choice of $\omega$ there is $m^{\prime} \in V(G)$ with $\frac{m}{\alpha} \leq m^{\prime}$, thus $\alpha<m<m^{\prime} \alpha$ contradicting $m^{\prime} \in V(G)$.

Let $W$ be the convex hull of $V(G)$ in $M\langle\omega, \alpha\rangle$. Since $\alpha \cdot \omega$ realizes $G^{+}$the cut $r:=\alpha \cdot W^{+}$ of $M\langle\omega, \alpha\rangle$ extends $p_{2}$. Obviously $V(r)=W$, hence $V(r)^{+}$extends $p_{1}$. By (2.15), there are $N \succ M\langle\alpha, \omega\rangle$ and an extension $q_{2}$ of $r$ on $N$ with $q_{2}=\hat{q}_{2}$, sign ${ }^{*} q_{2}=0$ such that $V\left(q_{2}\right)^{+}$ extends $V(r)^{+}$.

Let $A$ be the largest convex subset of $N$ with $A \cap M=V(G)$. Then $\omega \in A$ and it is straightforward to see that $A$ is a convex valuation ring of $N$. We take $q_{1}:=A^{+}$. Here the 
situation in a diagram, where a broken line indicates realization and a solid line indicates extension of cuts:

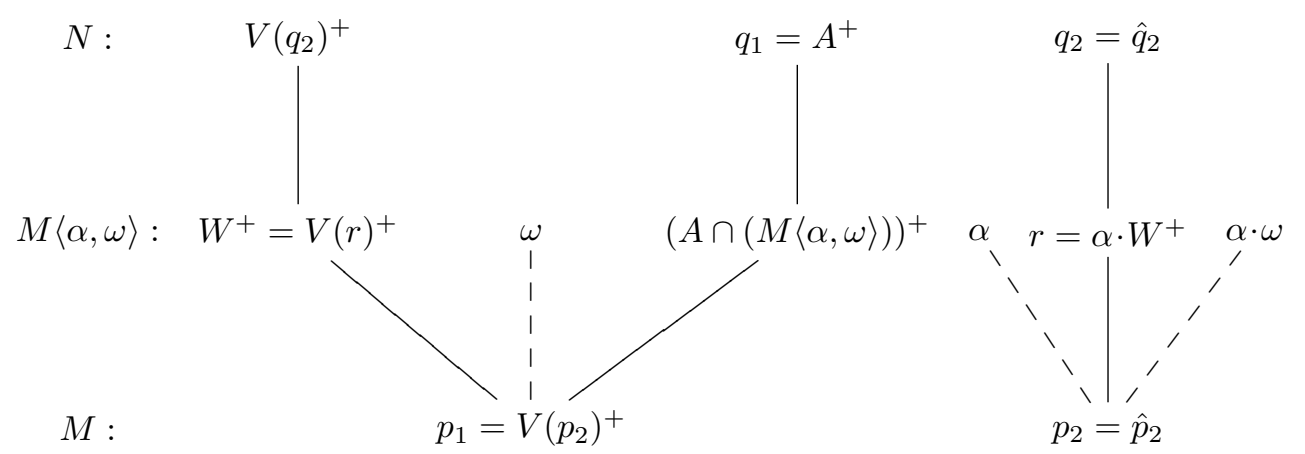

As $\operatorname{sign}^{*} q_{2}=0$ and $q_{2}=\hat{q}_{2},(2.13)$ says that $\left(q_{1}, q_{2}\right)$ is a box type.

Since $q_{1}$ extends $p_{1}$ and both cuts are upper edges of convex rings, $q_{1}$ is an heir of $p_{1}$. Since sign $^{*} p_{2}=0$ and $V\left(q_{2}\right)$ lies over $V\left(p_{2}\right)$ by construction, $q_{2}$ is an heir of $p_{2}$ (cf. (2.16)(iii)).

However, $\left(q_{1}, q_{2}\right)$ is not an heir of $\left(p_{1}, p_{2}\right)$, since $V\left(q_{2}\right)^{+}$is strictly less than $q_{1}$ : We have $\omega<q_{1}$ and $0<\alpha<q_{2}<\alpha \cdot \omega$ (as $q_{2}$ extends $r$ and $\alpha<r<\alpha \cdot \omega$ ), but there are no $m, m^{\prime} \in M$ with $m<p_{1}$ and $0<m^{\prime}<p_{2}<m \cdot m^{\prime}$ (as $p_{1}=V\left(p_{2}\right)^{+}$and $p_{2}=\hat{p}_{2}$ ). This finishes the example.

If $p$ is a cut of $M$ and $q$ is an heir of $p$ on $N \succ M$ then in general it is not the case that $q$ is an heir of $q\left\lceil M_{1}\right.$ for every $M \succ M_{1} \succ N$, even if $q\left\lceil M_{1}\right.$ is the unique extension of $p$ on $M_{1}$ and $\left(M, p^{L}\right)$ is an elementary substructure of $\left(N, q^{L}\right)$. For example with the aid of (2.15) we can produce the following situation: $p=\hat{p}$, $\operatorname{sign}^{*} p=0, M_{1}=M\langle\alpha\rangle$ with $\alpha \models V(p)^{+}$ and $q$ extends $p$ on $N \succ M\langle\alpha\rangle$ such that $q=\hat{q}$, $\operatorname{sign}^{*} q=0$ and $V(q) \cap M\langle\alpha\rangle$ is the convex hull of $V(p)$ in $M\langle\alpha\rangle$. Then $\left(M, p^{L}\right)$ is an elementary substructure of $\left(N, q^{L}\right)$ by (2.18)(i), in particular $q$ is an heir of $p$. $p$ is omitted in $M\langle\alpha\rangle$, since $\left(V(p)^{+}, p\right)$ is a box type by (4.2). But $q$ is not an heir of $q\lceil M\langle\alpha\rangle$, since $\alpha \cdot q>q$ (as $V(q)<\alpha)$ and $\alpha \cdot(q \uparrow M\langle\alpha\rangle)=q \uparrow M\langle\alpha\rangle$ by (2.4)(i) (note that $q\lceil M\langle\alpha\rangle$ is the unique extension of $p$ on $M\langle\alpha\rangle$ by construction).

The reason why $q$ is not an heir of $q \uparrow M_{1}$ in this example is that some member of box $(p)$ is realized in $M_{1}$. If this is not the case then we have

(4.4) Proposition. Let $M \prec N$ be models of $T$, let $p$ be a cut of $M$ and let $q$ be an extension of $p$ on $N$. Let $M \prec M_{1} \prec N$ be such that no member of $\operatorname{box}(p)$ is realized in $M_{1}$.

If $q$ is an heir of $p$ then $q$ is an heir of $q \uparrow M_{1}$.

Proof. We may assume that $p$ is not principal. If $p$ is dense, then $\operatorname{box}(p)=\left(p, 0^{+}\right)$. By assumption, $0^{+}$is omitted in $M_{1}$, in other words $M$ is archimedean in $M_{1}$. Since $p$ is omitted in $M_{1}$, the unique extension $q\left\lceil M_{1}\right.$ of $p$ on $M_{1}$ is again dense ((2.4)(i)). By (2.16)(ii), $q$ is an heir of $q \uparrow M_{1}$.

Thus we may assume that $p$ is neither principal nor dense. Then $\hat{p}$ is not principal. Since $\hat{p}$ is omitted in $M_{1}$ and $q_{1}:=q\left\lceil M_{1}\right.$ is an heir of $p, G\left(q_{1}\right)$ is the convex hull of $G(p)$ in $M_{1}$ and we get $G\left(q_{1}\right) \subseteq G(q)$. A similar argument shows that $V(q)$ lies over $V\left(q_{1}\right)$. If $\operatorname{sign} p=0$ then $\operatorname{sign} q_{1}=0$ and $q$ is an heir of $q_{1}$ by (2.16)(ii). Hence we may assume $\operatorname{sign} p \neq 0$ and by $(2.16)(\mathrm{i})$ we may also assume that $p=\hat{p}, q=\hat{q}$. If $\operatorname{sign}^{*} \hat{p}=0$, then $\operatorname{sign}^{*} \hat{q}_{1}=0$ and $q$ is an heir of $q_{1}$ by $(2.16)$ (iii). If $\operatorname{sign}^{*} \hat{p}$ is not 0 , say $\hat{p}=c \cdot V(p)^{+}$, then $\hat{q}_{1}=c \cdot V\left(q_{1}\right)^{+}$and $\hat{q}=c \cdot V(q)^{+}$, thus $q$ is an heir of $q_{1}$. 


\section{Two Methods PRODUCING BOX TYPES}

The first method allows to glue box types from cuts with different invariance rings:

(5.1) TheOrem. Let $M$ be a model of $T$ and let $\left(p_{1}, \ldots, p_{n}\right),\left(q_{1}, \ldots, q_{k}\right)$ be box types of $M$. If $V\left(q_{j}\right) \neq V\left(p_{i}\right)$ for all $i, j$, then $\left(p_{1}, \ldots, p_{n}, q_{1}, \ldots, q_{k}\right)$ is a box type.

Proof. The substantial part of the theorem is the case $n=k=1$ :

Claim. The theorem holds if $n=k=1$.

To see this we write $p=p_{1}, q=q_{1}$. Suppose $(p, q)$ is not a box type, i.e. $p \sim q$. We may assume that $V(q) \neq M$. Then $\left(M, p^{L}\right)$ defines $V(q) \neq V(p)$. If $p$ is principal or dense, then, as $q$ is realized in $M\langle\alpha\rangle, \alpha=p, q$ is principal or dense, too and $V(q)=M=V(p)$, a contradiction. Thus we may assume that $p, q$ both are neither dense nor definable and $V(p) \neq M$. Suppose $\operatorname{sign} p \neq 0$ and $\operatorname{sign}^{*} \hat{p} \neq 0$. Then $p^{L}$ is definable in $(M, V(p))$, which implies that $V(q)$ is definable in $(M, V(p))$. But this is impossible by $(2.12)$ : as $V(q) \neq V(p)$, $V(q)^{+}$can not be of the form $a+b \cdot V(p)^{+}$or $a+b \cdot \mathfrak{m}(p)^{+}$. Thus we have sign $p=0$ or $\operatorname{sign}^{*} \hat{p}=0$. We write $V:=V(q)$.

Let $\alpha$ be a realization of $p$, let $W$ be the convex hull of $V$ in $M\langle\alpha\rangle$ and let $p_{1}$ be the largest extension of $p$ on $M\langle\alpha\rangle$.

Subclaim. If $\operatorname{sign} p=0$, then $W$ is definable in $\left(M\langle\alpha\rangle, G\left(p_{1}\right)\right)$.

Proof. Firstly by (2.13), $\hat{p}_{1}$ is the unique extension of $\hat{p}$ on $M\langle\alpha\rangle$ and $V\left(p_{1}\right), W$ are the unique convex valuation rings of $M\langle\alpha\rangle$ lying over $V(p), V$, respectively. Moreover, by $(2.4)(\mathrm{iv}), \operatorname{sign} p_{1} \neq 0$ and by $(2.4)(\mathrm{v}), \operatorname{sign}^{*} \hat{p}_{1}=\operatorname{sign}^{*} \hat{p}$.

Case $1 . \operatorname{sign}^{*} \hat{p} \neq 0$.

We do the case $G(p)=a \cdot V(p)$ for some $a \in M$, the case $G(p)=a \cdot \mathfrak{m}(p)$ is similar. Since $p$ is not dense, $V(p)$ is proper and $\hat{p}$ is not principal. By (2.17) applied to our situation, the theory of $\left(M, V(p), G(p), Z^{*}, p^{L}\right)$ is model complete in the language $\mathscr{L}\left(\Theta, \mathscr{G}, \mathscr{Z}^{*}, \mathscr{D}\right)$, where $Z^{*}=\left\{b \in M^{>0} \mid b \cdot V(p)=G(p)\right\}$. Then, if $c$ is a new constant symbol, also the theory of $\left(M, V(p), G(p), p^{L}, a\right)$ in the language $\mathscr{L}(\Theta, \mathscr{G}, \mathscr{D}, c)$ is model complete (observe that $b \in Z^{*} \Leftrightarrow b>0$ and $\left.\frac{b}{a}, \frac{a}{b} \in V(p)\right)$. Let $\chi(x, \bar{u}, \bar{v})$ be a quantifier free formula in the language $\mathscr{L}(\Theta, \mathscr{G}, \mathscr{D}, c)$ and let $\bar{b} \in M^{\bar{v}}$ such that $V$ is defined by $\varphi(x):=\exists \bar{u} \chi(x, \bar{u}, \bar{b})$. By (2.16)(vi), $\left(V\left(p_{1}\right)^{+}, \hat{p}_{1}, p_{1}\right)$ is an heir of $\left(V(p)^{+}, \hat{p}, p\right)$, in other words $\left(M, V(p), G(p), p^{L}\right)$ is existentially closed in $\left(M\langle\alpha\rangle, V\left(p_{1}\right), G\left(p_{1}\right), p_{1}^{L}\right)$. Therefore the set $W_{0}$ defined by the formula $\varphi(x)$ in $\left(M\langle\alpha\rangle, V\left(p_{1}\right), G\left(p_{1}\right), p_{1}^{L}\right)$ intersects $M$ in $V$. Since $W$ is the unique convex valuation ring of $M\langle\alpha\rangle$ lying over $V, W$ is the convex hull of $W_{0}$. Thus $W$ is definable in $\left(M\langle\alpha\rangle, p_{1}^{L}\right)$. Since sign $p_{1} \neq 0, W$ is definable in $\left(M\langle\alpha\rangle, G\left(p_{1}\right)\right)$.

Case 2. $\operatorname{sign}^{*} \hat{p}=0$.

Similar to case 1 , we sketch it: By (2.18), the theory of $\left(M, V(p), G(p), p^{L}\right)$ is model complete. Hence there is a quantifier free formula $\chi(x, \bar{u}, \bar{v})$ in the language $\mathscr{L}(\Theta, \mathcal{G}, \mathcal{D})$ and $\bar{b} \in M^{\bar{v}}$ such hat $V$ is defined by $\exists \bar{u} \chi(x, \bar{u}, \bar{b})$. Similar to case $1,(2.16)(\mathrm{v})$ implies that $\left(M, V(p), G(p), p^{L}\right)$ is existentially closed in $\left(M\langle\alpha\rangle, V\left(p_{1}\right), G\left(p_{1}\right), p_{1}^{L}\right)$. As in case 1 , it follows that $W$ is definable in $\left(M\langle\alpha\rangle, G\left(p_{1}\right)\right)$. This finishes the proof of the subclaim.

Now, if $\operatorname{sign} p=0$, then using the subclaim we replace $M$ by $M\langle\alpha\rangle, V$ by $W$ and $p$ by $p_{1}$, and we obtain a cut $p$ with $\operatorname{sign} p \neq 0$ such that $\left(M, p^{L}\right)$ defines a proper convex valuation ring $V \neq V(p)$. Then we may also replace $p$ by $\hat{p}$. Hence we have a proper convex valuation 
ring $V$, definable in $(M, G)$ for a convex subgroup $G$ such that $V \neq V(G)$. By $(2.12), V$ is not definable in $(M, V(G))$. Since $V$ is definable in $(M, G), G^{+}$has multiplicative signature 0 , thus $G^{+} \not V(G)^{+}$. Let $\alpha$ be a realization of $G^{+}$, let $W$ be the convex hull of $V$ in $M\langle\alpha\rangle$ and let $H$ be the convex hull of $G$ in $M\langle\alpha\rangle$. Then $V(H)$ is the unique convex valuation ring of $M\langle\alpha\rangle$ lying over $V(G)$ and $W \neq V(H)$. By (2.12) again, $W$ is not definable in $(M\langle\alpha\rangle, V(H))$.

On the other hand the theory of $(M, V(G), G)$ is model complete by $(2.18)(\mathrm{i})$, hence there is a quantifier free formula $\chi(x, \bar{u}, \bar{v})$ in the language $\mathscr{L}(\Theta, \mathcal{G})$ and $\bar{a} \in M^{\bar{v}}$ such hat $V$ is defined by $\exists \bar{u} \chi(x, \bar{u}, \bar{a})$. By $(2.16)$ (iii), $\left(V(H)^{+}, H^{+}\right)$is an heir of $\left(V(G)^{+}, G^{+}\right)$, hence $(M, V(G), G)$ is existentially closed in $(M\langle\alpha\rangle, V(H), H)$ and as in the proof of case 1 of the subclaim, we get that $W$ is definable in $(M\langle\alpha\rangle, V(H))$, a contradiction. This finishes the proof of the claim, i.e. the theorem holds if $n=k=1$.

Next we prove the theorem in the case $k=1$. We write $q=q_{1}$ and do an induction on $n$, where $n=1$ holds by the claim. For the induction step, take a realization $\alpha$ of $p_{1}$. Since $\left(p_{1}, \ldots, p_{n}\right)$ is a box type, $p_{i}$ has a unique extension $p_{i}^{\prime}$ on $M\langle\alpha\rangle(2 \leq i \leq n)$. By (2.4), applied to $p_{i}$ and then multiplicatively to $\hat{p}_{i}, V\left(p_{i}^{\prime}\right)$ lies over $V\left(p_{i}\right)$ for all $i \geq 2$. From the case $n=1$ we know that $q$ is not realized in $M\langle\alpha\rangle$, hence $q$ has a unique extension $q^{\prime}$ on $M\langle\alpha\rangle$, too. Again $V\left(q^{\prime}\right)$ lies over $V(q)$. It follows that $V\left(q^{\prime}\right) \neq V\left(p_{2}^{\prime}\right), \ldots, V\left(p_{n}^{\prime}\right)$. By $(3.4)$, $\left(p_{2}^{\prime}, \ldots, p_{n}^{\prime}\right)$ is a box type and we may apply the induction hypothesis. Hence $\left(p_{2}^{\prime}, \ldots, p_{n}^{\prime}, q^{\prime}\right)$ is a box type and by (3.4) again, $\left(p_{1}, \ldots, p_{n}, q\right)$ is a box type, too.

This shows the theorem in the case $k=1$. If $k>1$, then inductively we know that $\left(p_{1}, \ldots, p_{n}, q_{1}, \ldots, q_{k-1}\right)$ is a box type. Take a realization $\bar{\alpha}$ of $\left(q_{1}, \ldots, q_{k-1}\right)$ and let $N:=M\langle\bar{\alpha}\rangle$. Let $p_{1}^{\prime}, \ldots, p_{n}^{\prime}, q_{k}^{\prime}$ be the unique extensions of $p_{1}, \ldots, p_{n}, q_{k}$ on $N$ respectively. By (2.4), applied to $p_{i}, q_{k}$ and then multiplicatively to $\hat{p}_{i}$ and $\hat{q}_{k}, V\left(p_{i}^{\prime}\right)$ lies over $V\left(p_{i}\right)$ and $V\left(q_{k}^{\prime}\right)$ lies over $V\left(q_{k}\right)$. Hence we may apply the case $k=1$ and we get that $\left(p_{1}^{\prime}, \ldots, p_{n}^{\prime}, q_{k}^{\prime}\right)$ is a box type. By $(3.4),\left(p_{1}, \ldots, p_{n}, q_{1}, \ldots, q_{k}\right)$ is a box type.

(5.2) Corollary. Let $M$ be a model of $T$ and let $p_{1}, \ldots, p_{n}$ be cuts of $M$ with $V\left(p_{i}\right) \neq V\left(p_{j}\right)$ $(1 \leq i \neq j \leq n)$. Then $\left(p_{1}, \ldots, p_{n}\right)$ is a box type.

Proof. By induction on $n$ from (5.1).

(5.3) Corollary. Let $M$ be a model of $T$ and let $\left(p_{1}, \ldots, p_{n}\right)$ be a box type of $M$. Let $f: M^{n} \longrightarrow M$ be $M$-definable and let $q:=f\left(p_{1}, \ldots, p_{n}\right)$. Suppose $V\left(p_{k+1}\right), \ldots, V\left(p_{n}\right) \neq V(q)$. Then there is an $M$-definable map $g: M^{k} \longrightarrow M$ with $q=g\left(p_{1}, \ldots, p_{k}\right)$.

ProOF. It is enough to show that $q$ is realized in $N:=M\langle\bar{\alpha}\rangle$ for some realization $\bar{\alpha}$ of $\left(p_{1}, \ldots, p_{k}\right)$. Since $\left(p_{1}, \ldots, p_{n}\right)$ is a box type the cuts $p_{k+1}, \ldots, p_{n}$ have unique extensions $p_{k+1}^{\prime}, \ldots, p_{n}^{\prime}$ on $N$, respectively. By (2.4), applied to $p_{i}$ and then multiplicatively to $\hat{p}_{i}, V\left(p_{i}^{\prime}\right)$ lies over $V\left(p_{i}\right)(i>k)$. If $q$ is omitted in $N$, then also $q$ has a unique extension $q^{\prime}$ on $N$ and again, $V\left(q^{\prime}\right)$ lies over $V(q)$. Thus by assumption $V\left(q^{\prime}\right) \neq V\left(p_{k+1}^{\prime}\right), \ldots, V\left(p_{n}^{\prime}\right)$. By (3.4), $\left(p_{k+1}^{\prime}, \ldots, p_{n}^{\prime}\right)$ is a box type and by $(5.1)$, also $\left(p_{k+1}^{\prime}, \ldots, p_{n}^{\prime}, q^{\prime}\right)$ is a box type. By (3.4), again, $\left(p_{1}, \ldots p_{n}, q\right)$ is a box type which contradicts the existence of $f$.

The second method to produce box types allows to glue box types from cuts with distinct signatures: 
(5.4) Proposition. Let $M$ be a model of $T$ and let $p_{1}, \ldots, p_{n}, q_{1}, \ldots, q_{m}, r_{1}, \ldots, r_{l}$ be cuts of M. Suppose

(i) $\left(q_{1}, \ldots, q_{m}\right)$ and $\left(p_{1}, \ldots, p_{n}\right)$ are box types.

(ii) $\operatorname{sign} r_{i} \neq 0$, $\operatorname{sign}^{*} \hat{r}_{i} \neq 0$ and $V\left(r_{i}\right) \neq V\left(r_{j}\right)(1 \leq i \neq j \leq l)$.

(iii) $\operatorname{sign} q_{i} \neq 0$ and $\operatorname{sign}^{*} \hat{q}_{i}=0(1 \leq i \leq m)$.

(iv) $\operatorname{sign} p_{i}=0(1 \leq i \leq n)$.

Then $\left(r_{1}, \ldots, r_{l}, q_{1}, \ldots, q_{m}, p_{1}, \ldots, p_{n}\right)$ is a box type.

Proof. Of course we may assume that $r_{i}=V\left(r_{i}\right)^{+}$and $q_{i}=\hat{q}_{i}$. By $(5.2),\left(r_{1}, \ldots, r_{l}\right)$ is a box type.

Claim 1: $\left(r_{1}, \ldots, r_{l}, q_{1}\right)$ is a box type.

We prove this by induction on $l$. Note that by (2.13)(ii), if $G$ is a convex subgroup of $(M,+, \leq)$ and sign ${ }^{*} G^{+}=0$, then $G^{+}$is not equivalent to $V^{+}$if $V$ is a convex valuation ring of $M$. In particular we know claim 1 for $l=1$. Suppose we know that $\left(r_{1}, \ldots, r_{l-1}, q_{1}\right)$ is a box type. Let $\alpha_{1}, \ldots, \alpha_{l-1}$ be realizations of $r_{1}, \ldots, r_{l-1}$. Then $q_{1}$ and $r_{l}$ are omitted in $M\left\langle\alpha_{1}, \ldots, \alpha_{l-1}\right\rangle$. Hence the unique extension $q^{\prime}$ of $q_{1}$ on $M\left\langle\alpha_{1}, \ldots, \alpha_{l-1}\right\rangle$ has again multiplicative signature 0 . Therefore $q^{\prime}$ is not equivalent to the unique extension $r^{\prime}$ of $r_{l}$ on $M\left\langle\alpha_{1}, \ldots, \alpha_{l-1}\right\rangle$. By (3.4), this shows that $\left(r_{1}, \ldots, r_{l}, q_{1}\right)$ is a box type.

Claim 2: $\left(r_{1}, \ldots, r_{l}, q_{1}, \ldots, q_{m}\right)$ is a box type.

We prove this by induction on $m$. The case $m=1$ has been done in claim 1. Suppose we know that $\left(r_{1}, \ldots, r_{l}, q_{1}, \ldots, q_{m-1}\right)$ is a box type. Let $\beta_{1}, \ldots, \beta_{m-1}$ be realizations of $q_{1}, \ldots, q_{m-1}$ respectively. Then $q_{m}$ and each $r_{i}$ is omitted in $M\left\langle\beta_{1}, \ldots, \beta_{m-1}\right\rangle$. In particular the unique extension $q^{\prime}$ of $q_{m}$ on $M\left\langle\beta_{1}, \ldots, \beta_{m-1}\right\rangle$ has multiplicative signature 0 . If $r_{1}^{\prime}, \ldots, r_{l}^{\prime}$ denote the unique extensions of $r_{1}, \ldots, r_{l}$ on $M\left\langle\beta_{1}, \ldots, \beta_{m-1}\right\rangle$ respectively, then $\left(r_{1}^{\prime}, \ldots, r_{l}^{\prime}\right)$ is a box type. By claim 1 we know that $\left(r_{1}^{\prime}, \ldots, r_{l}^{\prime}, q^{\prime}\right)$ is a box type and by $(3.4)$, this gives claim 2 .

Claim 3: Suppose $G_{1}, \ldots, G_{k}$ are convex subgroups of $(M,+, \leq)$ such that $\left(G_{1}^{+}, \ldots, G_{k}^{+}\right)$is a box type. If $p$ is a cut of $M$ with $\operatorname{sign} p=0$, then $\left(G_{1}^{+}, \ldots, G_{k}^{+}, p\right)$ is a box type.

We prove this by induction on $k$. If $k=1$, this holds true by $(2.13)(\mathrm{i})$. Suppose we know that $\left(G_{1}^{+}, \ldots, G_{k-1}^{+}, p\right)$ is a box type. Let $\alpha_{1}, \ldots, \alpha_{k-1}$ be realizations of $G_{1}^{+}, \ldots, G_{k-1}^{+}$ respectively. Then $p$ and $G_{k}^{+}$are omitted in $M\left\langle\alpha_{1}, \ldots, \alpha_{k-1}\right\rangle$, hence the unique extension $p^{\prime}$ of $p$ on $M\left\langle\alpha_{1}, \ldots, \alpha_{k-1}\right\rangle$ has again signature 0 . Thus $H^{+}$and $p^{\prime}$ are not equivalent, where $H$ denotes the convex hull of $G_{k}$ in $M\left\langle\alpha_{1}, \ldots, \alpha_{k-1}\right\rangle$. By (3.4), this proves claim 3.

Proof of the proposition. We prove that $\left(r_{1}, \ldots, r_{l}, q_{1}, \ldots, q_{m}, p_{1}, \ldots, p_{n}\right)$ is a box type by induction on $n$. If $n=1$, then we know this from claim 2 and claim 3 . Suppose we know that $\left(r_{1}, \ldots, r_{l}, q_{1}, \ldots, q_{m}, p_{1}, \ldots, p_{n-1}\right)$ is a box type. Let $\alpha_{1}, \ldots, \alpha_{n-1}$ be realizations of $p_{1}, \ldots, p_{n-1}$, respectively. By assumption, $p_{n}$ has a unique extension $p^{\prime}$ on $M\left\langle\alpha_{1}, \ldots, \alpha_{n-1}\right\rangle$, which is again of signature 0 . Let $r_{1}^{\prime}, \ldots, r_{l}^{\prime}, q_{1}^{\prime}, \ldots, q_{m}^{\prime}$ denote the unique extensions of $r_{1}, \ldots, r_{l}, q_{1}, \ldots, q_{m}$ on $M\left\langle\alpha_{1}, \ldots, \alpha_{n-1}\right\rangle$ respectively. Then $\left(r_{1}^{\prime}, \ldots, r_{l}^{\prime}, q_{1}^{\prime}, \ldots, q_{m}^{\prime}\right)$ is a box type and by claim 3 we know that $\left(r_{1}^{\prime}, \ldots, r_{l}^{\prime}, q_{1}^{\prime}, \ldots, q_{m}^{\prime}, p^{\prime}\right)$ is a box type. By (3.4) again, this proves the proposition.

\section{HeIRS OF BOX TYPES}

In (4.3) we have seen that $\left(q_{1}, \ldots, q_{n}\right)$ does not need to be an heir of $\left(p_{1}, \ldots, p_{n}\right)$ if $q_{i}$ is an heir of $p_{i}$ for each $i$. Our first aim is $(6.2)$, which shows that $\left(q_{1}, \ldots, q_{n}\right)$ is an heir of $\left(p_{1}, \ldots, p_{n}\right)$ under the additional assumption $V\left(p_{i}\right) \neq V\left(p_{j}\right)(i \neq j)$. 
(6.1) Lemma. Let $p, p_{1}, \ldots, p_{n}$ be $n+1$ cuts of $M=T$ and let $N \succ M$ such that none of the $p_{i}$ is realized in $N$. Let $q_{i}$ be the unique extension of $p_{i}$ to $N$. Then for every heir $q$ of $p$ on $N,\left(q, q_{1}, \ldots, q_{n}\right)$ is an heir of $\left(p, p_{1}, \ldots, p_{n}\right)$.

Proof. Let $\bar{p}$ be an $(n+1)$-type containing $p\left(x_{0}\right) \cup p_{1}\left(x_{1}\right) \cup \ldots \cup p_{n}\left(x_{n}\right)$. Since $q$ is an heir of $p$, a standard compactness argument shows that there is an heir $\bar{q}$ of $\bar{p}$ on $N$ which contains $q\left(x_{0}\right)$. Since $q_{i}$ is the unique extension of $p_{i}, p_{i}\left(x_{i}\right) \subseteq \bar{p}$ implies $q_{i}\left(x_{i}\right) \subseteq \bar{q}$. Hence $\left(q, q_{1}, \ldots, q_{n}\right)$ is an heir of $\left(p, p_{1}, \ldots, p_{n}\right)$.

(6.2) Proposition. Let $M$ be a model of $T$ and let $p_{1}, \ldots, p_{k}$ be cuts of $M$ with $V\left(p_{i}\right) \neq V\left(p_{j}\right)$ $(1 \leq i \neq j \leq k)$. For every $N \succ M$ and all heirs $q_{i}$ of $p_{i}$ on $N(1 \leq i \leq k),\left(q_{1}, \ldots, q_{k}\right)$ is an heir of $\left(p_{1}, \ldots, p_{k}\right)$.

Proof. Firstly, by $(5.2),\left(p_{1}, \ldots, p_{k}\right)$ is a box type. We prove the proposition by induction on $k$. There is nothing to do if $k=1$. Assume we have proved the proposition for $k$ cuts; Let $p_{0}, p_{1}, \ldots, p_{k} \in S_{1}(M)$ with mutually distinct invariance rings, and let $q_{i}$ be an heir of $p_{i}$ on $N(0 \leq i \leq k)$. By (2.6), we have to show the following:

If $\bar{\alpha}, \bar{\beta} \in N^{k}, \gamma, \delta \in N$ and $\varphi(u, v, \bar{w}, \bar{z})$ is a formula in the language $\mathscr{L}(M)$ with $(2 k+2)$ free variables, such that

$$
\gamma<q_{0}<\delta, \alpha_{i}<q_{i}<\beta_{i}(1 \leq i \leq k) \text { and } N \models \varphi(\gamma, \delta, \bar{\alpha}, \bar{\beta}),
$$

then there are $\bar{a}, \bar{b} \in M^{k}, c, d \in M$ such that

$$
c<p_{0}<d, a_{i}<p_{i}<b_{i}(1 \leq i \leq k) \text { and } M \models \varphi(c, d, \bar{a}, \bar{b}) .
$$

We shall assume that $\delta$ and $\gamma$ are realizations of $p_{0}$. The other cases are easier and can be treated in a similar way. Let $p_{i}^{\prime}$ be the restriction of $q_{i}$ to $M\langle\gamma\rangle$. Since $\left(p_{0}, p_{1}, \ldots, p_{k}\right)$ is a box type, $p_{i}^{\prime}$ is the unique extension of $p_{i}$ on $M\langle\gamma\rangle$ for all $i \geq 1$. Since $q_{0}$ is an heir of $p_{0}$ and $\gamma<q_{0}, p_{0}^{\prime}$ is the largest extension of $p_{0}$ on $M\langle\gamma\rangle$. In each case we know that $V\left(p_{i}^{\prime}\right)$ lies over $V\left(p_{i}\right)$, in particular $V\left(p_{i}^{\prime}\right) \neq V\left(p_{j}^{\prime}\right)(0 \leq i \neq j \leq k)$. By $(5.2),\left(p_{0}^{\prime}, p_{1}^{\prime}, \ldots, p_{k}^{\prime}\right)$ is again a box type. For $i \geq 1$, the invariance ring of each member of $\operatorname{box}\left(p_{i}\right)$ is $V\left(p_{i}\right)$ again. As $\gamma \models p_{0}$ and $V\left(p_{0}\right) \neq V\left(p_{i}\right),(5.1)$ implies that no member of $\operatorname{box}\left(p_{i}\right)$ is realized in $M\langle\gamma\rangle$. It follows from (4.4) that $q_{i}$ is an heir of $p_{i}^{\prime}$ for all $i \geq 1$.

Since $p_{0}^{\prime}$ is the largest extension of $p_{0}$ on $M\langle\gamma\rangle$ and $\delta>q_{0}$ is a realization of $p_{0}, \delta$ also realizes $p_{0}^{\prime}$. Since $\left(p_{0}^{\prime}, p_{1}^{\prime}, \ldots, p_{k}^{\prime}\right)$ is a box type, $p_{i}^{\prime}$ is omitted in $M\langle\gamma, \delta\rangle$ for all $i \geq 1$. Consequently for each $i \geq 1$, the invariance ring of the unique extension $p_{i}^{\prime \prime}$ of $p_{i}^{\prime}$ on $M\langle\gamma, \delta\rangle$ lies over $V\left(p_{i}^{\prime}\right)$, in particular $V\left(p_{i}^{\prime \prime}\right) \neq V\left(p_{j}^{\prime \prime}\right)(1 \leq i \neq j \leq k)$. Moreover, by (5.2), every cut of $M\langle\gamma\rangle$ realized in $M\langle\gamma, \delta\rangle$ has invariance ring $V\left(p_{0}^{\prime}\right)$. By (4.4), applied to $p_{i}^{\prime}, q_{i}$ and $M\langle\gamma, \delta\rangle, q_{i}$ is an heir of $p_{i}^{\prime \prime}$ for all $i \geq 1$. By the induction hypothesis applied to $\left(p_{1}^{\prime \prime}, \ldots, p_{k}^{\prime \prime}\right)$, $\left(q_{1}, \ldots, q_{k}\right)$ is an heir of $\left(p_{1}^{\prime \prime}, \ldots, p_{k}^{\prime \prime}\right)$. Hence there are $\bar{\rho}, \bar{\tau} \in M\langle\gamma, \delta\rangle^{k}$ such that

$$
\gamma<p_{0}^{\prime \prime}<\delta, \rho_{i}<p_{i}^{\prime \prime}<\tau_{i}(1 \leq i \leq k) \text { and } N=\varphi(\gamma, \delta, \bar{\rho}, \bar{\tau}),
$$

where $p_{0}^{\prime \prime}$ is the restriction of $q_{0}$ to $M\langle\gamma, \delta\rangle$. Since $p_{i}$ is omitted in $M\langle\gamma, \delta\rangle$ for all $i \geq 1$ and $p_{0}^{\prime \prime}$ is an heir of $p_{0}$ we may apply $(6.1)$, thus $\left(p_{0}^{\prime \prime}, p_{1}^{\prime \prime}, \ldots, p_{k}^{\prime \prime}\right)$ is an heir of $\left(p_{0}, p_{1}, \ldots, p_{n}\right)$. Hence there are $\bar{a}, \bar{b} \in M^{k}, c, d \in M$ as claimed.

(6.3) Corollary. Let $\mathscr{L}_{\text {convex }, l}$ be the language $\mathscr{L}$ together with $l$ new unary relation symbols $\Theta_{1}, \ldots, \Theta_{l}$ and let $T_{\text {convex }, l}$ be the $\mathscr{L}_{\text {convex }, l}$-theory $T$ together with the statement that $\Theta_{1}, \ldots, \theta_{l}$ are proper, convex valuation rings such that $\Theta_{1} \varsubsetneqq \ldots \varsubsetneqq \Theta_{l}$. 
Then $T_{\text {convex,l }}$ is complete. If $T$ is model complete, then $T_{\text {convex,l }}$ is model complete. If $T$ has quantifier elimination and a universal system of axioms, then $T_{\text {convex,l }}$ has quantifier elimination.

Proof. The completeness of $T_{\text {convex }, l}$ follows from the quantifier elimination of $T_{\text {convex }, l}^{d f}$, since this theory has the prime structure $(\mathscr{P},(\underbrace{\mathscr{P}, \ldots, \mathscr{P}}_{l \text {-times }}))$, where $\mathscr{P}$ is the prime model of $T^{d f}$. Model completeness of $T_{\text {convex }, l}$ follows from (6.2) and the Robinson test for model completeness, provided $T$ is already model complete.

Now assume $T$ has quantifier elimination and a universal system of axioms. Let $\mathscr{M}=$ $\left(M, V_{1}, \ldots, V_{l}\right)$ and $\mathscr{N}=\left(M, W_{1}, \ldots, W_{l}\right)$ be models of $T_{\text {convex }, l}$ and let $\mathscr{A}=\left(A, B_{1}, \ldots, B_{l}\right)$ be a common substructure of $\mathscr{M}$ and $\mathscr{N}$. We prove that $\mathscr{M}$ and $\mathscr{N}$ are elementary equivalent over $A$. This gives quantifier elimination of $T_{\text {convex }, l}$. Since $T$ has quantifier elimination and a universal system of axioms, we know that $A \prec M, N$. Let $V_{l+1}:=M, W_{l+1}:=N$ and $B_{l+1}:=A$. If $B_{i} \neq B_{i+1}$ for all $i \leq l$, then $\mathscr{A}$ is a model of $T_{\text {convex }, l}$ and by model completeness of this theory we are done.

Otherwise, let $i \in\{1, \ldots, l\}$ be minimal with $B_{i}=B_{i+1}$ and choose $m \in M, n \in N$ with $V_{i}<m \in V_{i+1}$ and $W_{i}<n \in W_{i+1}$. Then the cut of $m$ over $A$ is the same as the cut of $n$ over $A$ and there is a unique $\mathscr{L}$-isomorphism $\varphi: A\langle m\rangle \longrightarrow A\langle n\rangle$ over $A$ mapping $m$ to $n$. We claim that $\varphi$ maps $A\langle m\rangle \cap V_{j}$ to $A\langle n\rangle \cap W_{j}$ for every $j \in\{1, \ldots, l+1\}$. To see this it is enough to show that $A\langle m\rangle \cap V_{j}$ is the convex hull of $B_{j}$ in $A\langle m\rangle$ if $j \leq i$ and $A\langle m\rangle \cap V_{j}$ is the largest convex valuation ring of $A\langle m\rangle$ lying over $B_{j}$ if $j>i$; applying this fact also to $A\langle n\rangle \cap W_{j}$ proves $\varphi\left(A\langle m\rangle \cap V_{j}\right)=A\langle n\rangle \cap W_{j}$.

If $B_{j} \neq B_{i}$, then - as $m \models B_{i}^{+} \not B_{j}^{+}-B_{j}^{+}$is omitted in $A\langle m\rangle$ and we are done. So we may assume that $B_{j}=B_{i}$, hence $i \leq j$. Since $\operatorname{dim}_{A} A\langle m\rangle=1$, there are exactly two valuation rings of $A\langle m\rangle$ lying over $B_{i}$. By choice of $m$, one is $A\langle m\rangle \cap V_{i}$ and the other one is $A\langle m\rangle \cap V_{i+1}$. Hence if $j=i$, then $A\langle m\rangle \cap V_{j}$ is the convex hull of $B_{j}$ and if $j>i$, then $A\langle m\rangle \cap V_{j}$ is the largest convex valuation ring of $A\langle m\rangle$ lying over $B_{j}$ as claimed.

This shows that $\varphi$ respects the $\mathscr{L}_{\text {convex }, l}$-structure induced from $\mathscr{M}, \mathscr{N}$ on $A\langle m\rangle, A\langle n\rangle$, respectively. We now identify $A\langle m\rangle$ with $A\langle n\rangle$ via $\varphi$ and repeat the argument above until we have reached a common substructure $\mathscr{S}$ of $\mathscr{M}$ and $\mathscr{N}$, which is a model of $T_{\text {convex,l }}$, too. By model completeness we know that $\mathscr{M}$ and $\mathscr{N}$ are elementary equivalent over $\mathscr{S}$, in particular $\mathscr{M}$ and $\mathscr{N}$ are elementary equivalent over $A$.

We now turn to the main result of this paper (6.6), which allows in principal to detect all heirs of box types.

(6.4) Definition. If $p_{1}, \ldots, p_{n}, r \in S_{1}(M)$ and $f: M^{n} \longrightarrow M$ is an $M$-definable map, we say $f\left(p_{1}, \ldots, p_{n}\right) \leq r$ if and only if $f(q) \leq r$ for each $q \in S_{n}(M)$ with projections $p_{1}, \ldots, p_{n}$. We say $f\left(p_{1}, \ldots, p_{n}\right)=r$ if $f\left(p_{1}, \ldots, p_{n}\right) \leq r$ and $f\left(p_{1}, \ldots, p_{n}\right) \geq r$.

(6.5) Remark. Let $X$ denote the set of all $n$-types $q \in S_{n}(M)$ with projections $p r_{i} q=p_{i} \notin$ $M(1 \leq i \leq n)$. Then $f(q) \leq r$ for all $q \in X$ if and only if for each $a \in M, a>r$ there is some open box $B=\prod\left(c_{i}, d_{i}\right)$ with $c_{i}<p_{i}<d_{i}(1 \leq i \leq n)$ such that $f(\bar{b})<a(\bar{b} \in B)$.

Thus $f\left(p_{1}, \ldots, p_{n}\right)=r$ if and only if the sets $\left\langle\right.$ convex hull $\left.l_{M} f(B)\right\rangle$, where $B$ runs through the open boxes containing $\left(p_{1}, \ldots, p_{n}\right)$, form a basis for the neighborhood of $r$ in the topology of $S_{1}(M)$.

ProOF. $X$ is the intersection of all $\langle B\rangle$, where $B$ runs through the open boxes $B=\prod\left(c_{i}, d_{i}\right)$ with $c_{i}<p_{i}<d_{i}(1 \leq i \leq n)$. By compactness we get the assertion. 
(6.6) Theorem. (Heirs of box types)

Let $M \prec N$ be models of $T$. Let $V_{1}, \ldots, V_{l}$ be mutually distinct, proper, convex valuation rings of $M$, and let $W_{1}, \ldots, W_{l}$ be convex valuation rings of $N$ lying over $V_{1}, \ldots, V_{l}$, respectively. Let $G_{1}, \ldots, G_{m}$ be convex subgroups of $(M,+, \leq)$ and let $H_{1}, \ldots, H_{m}$ be convex subgroups of $(N,+, \leq)$ with $G_{i}=M \cap H_{i}(1 \leq i \leq m)$. Furthermore, let $p_{1}, \ldots, p_{n}$ be cuts of $M$ and let $q_{1}, \ldots, q_{n}$ be extensions of $p_{1}, \ldots, p_{n}$ on $N$, respectively. The cases $n=0$ or $m=0$ or $l=0$ are not excluded. Suppose the following conditions hold:

(i) $\operatorname{sign} p_{i}=0(1 \leq i \leq n)$ and $\operatorname{sign}^{*} G_{i}^{+}=0(1 \leq i \leq m)$.

(ii) $\left(p_{1}, \ldots, p_{n}\right)$ and $\left(G_{1}^{+}, \ldots, G_{m}^{+}\right)$are box types.

(iii) For each $p_{i}$, which is not dense there is an $M$-definable map $f: M^{m+l} \longrightarrow M$ with $f\left(V_{1}^{+}, \ldots, V_{l}^{+}, G_{1}^{+}, \ldots, G_{m}^{+}\right)=\hat{p}_{i}$ and $f\left(W_{1}^{+}, \ldots, W_{l}^{+}, H_{1}^{+}, \ldots, H_{m}^{+}\right) \leq \hat{q}_{i}$.

Observe that this condition is void if $n=0$.

(iv) For all $i \in\{1, \ldots, m\}$ we have $V\left(G_{i}\right) \subseteq V\left(H_{i}\right)$.

(v) For all $i \in\{1, \ldots, m\}, j \in\{1, \ldots, l\}$, if $V_{j}=V\left(G_{i}\right)$, then $W_{j} \subseteq V\left(H_{i}\right)$.

Then $\left(W_{1}^{+}, \ldots, W_{l}^{+}, H_{1}^{+}, \ldots, H_{m}^{+}, q_{1}, \ldots, q_{n}\right)$ is an heir of $\left(V_{1}^{+}, \ldots, V_{l}^{+}, G_{1}^{+}, \ldots, G_{m}^{+}, p_{1}, \ldots, p_{n}\right)$, in other words: $\left(M, V_{1}, \ldots, V_{l}, G_{1}, \ldots, G_{m}, p_{1}^{L}, \ldots, p_{n}^{L}\right)$ is existentially closed in $\left(N, W_{1}, \ldots, W_{l}, H_{1}, \ldots, H_{m}, q_{1}^{L}, \ldots, q_{n}^{L}\right)$.

Before proving this, we give some remarks and explanations of the statement.

(6.7) Remarks.

1. Items (iii), (iv) and (v) formulate conditions, which compare the given sequences of cuts

$$
\left(V_{1}^{+}, \ldots, V_{l}^{+}, G_{1}^{+}, \ldots, G_{m}^{+}, p_{1}, \ldots, p_{n}\right) \text { and }\left(W_{1}^{+}, \ldots, W_{l}^{+}, H_{1}^{+}, \ldots, H_{m}^{+}, q_{1}, \ldots, q_{n}\right),
$$

whereas (i) and (ii) do not compare these sequences. The comparison is of the form:

"whenever the cuts $V_{1}^{+}, \ldots, V_{l}^{+}, G_{1}^{+}, \ldots, G_{m}^{+}, p_{1}, \ldots, p_{n}$ together with their invariance groups and invariance rings are in a certain configuration, then the cuts $W_{1}^{+}, \ldots, W_{l}^{+}, H_{1}^{+}, \ldots, H_{m}^{+}, q_{1}, \ldots, q_{n}$ together with their invariance groups and invariance rings are in some other configuration".

E.g. an instance of (iii) might say: "if $G_{1}^{+}=\hat{p}_{1}$ then $H_{1}^{+} \leq \hat{q}_{1}$ ".

2. Under assumptions (i) and (ii) and if there are $M$-definable functions $f_{i}$ with

$$
f_{i}\left(V_{1}^{+}, \ldots, V_{l}^{+}, G_{1}^{+}, \ldots, G_{m}^{+}\right)=\hat{p}_{i}
$$

for every nondense $p_{i}$, then it is not difficult to see that every heir of

$$
p:=\left(V_{1}^{+}, \ldots, V_{l}^{+}, G_{1}^{+}, \ldots, G_{m}^{+}, p_{1}, \ldots, p_{n}\right),
$$

in the sense of definition (2.5), must fulfill all requirements of (6.6). Hence Theorem (6.6) gives a complete characterization of the heirs of $p$ in the sense of definition (2.5).

The determination of the complete types that are heirs of $p$ is not answered by (6.6).

3. Every expansion of $M \models T$ by a finite set $\mathscr{C}$ of convex subsets of $M$ is parametrically interdefinable with an expansion of the form $\left(M, V_{1}, \ldots, V_{l}, G_{1}, \ldots, G_{m}, p_{1}^{L}, \ldots, p_{n}^{L}\right)$ such that $\left(V_{1}^{+}, \ldots, V_{l}^{+}, G_{1}^{+}, \ldots, G_{m}^{+}, p_{1}, \ldots, p_{n}\right)$ satisfies (i), (ii) of (6.6) and such that there are functions $f_{i}$ with $f_{i}\left(V_{1}^{+}, \ldots, V_{l}^{+}, G_{1}^{+}, \ldots, G_{m}^{+}\right)=\hat{p}_{i}$ for every nondense $p_{i}$. This is proved in (7.6) below, where the functions $f_{i}$ may also be chosen to be 0 -definable.

The remarks above show that the theorem - in principal - characterizes the heirs of a box type in terms of the location of the invariance groups and the invariance rings of the involved cuts. 
4. I want to point out that the cases $n=0, m=0$ or $l=0$ are a priori not excluded and we do not demand that each $G_{i}$ or each $V_{i}$ is definable in $\left(M, p_{1}^{L}, \ldots, p_{n}^{L}\right)$; in particular, the invariance group of $p_{i}$ might have nothing to do with any $G_{j}$. Furthermore, the cuts $q_{i}$ may be principal and the groups $H_{i}$ may have multiplicative signature $\neq 0$.

ProOF OF (6.6):

We first prove by induction on $m$, starting with $m=0$, that $\left(M, V_{1}, \ldots, V_{l}, G_{1}, \ldots, G_{m}\right)$ is existentially closed in $\left(N, W_{1}, \ldots, W_{l}, H_{1}, \ldots, H_{m}\right)$. The case $m=0$ holds since by (6.2) we know that $\left(M, V_{1}, \ldots, V_{l}\right)$ is existentially closed in $\left(N, W_{1}, \ldots, W_{l}\right)$. For the induction step we write $G=G_{m+1}, H=H_{m+1}$. If $V(G)$ is not one of the $V_{i}$ 's, we add $V(G)$ to the sequence $\left(V_{1}, \ldots, V_{l}\right)$ and $V(H)$ to the sequence $\left(W_{1}, \ldots, W_{l}\right)$. This certainly preserves our assumptions. Hence we may assume that $V_{l}=V(G)$. By assumption we have $W_{l} \subseteq V(H)$.

Claim A. If $\tau \in N$ is a realization of $G^{+}$and $K:=M\langle\tau\rangle$, then

(a) $\left(K, W_{1} \cap K, \ldots ., W_{l} \cap K, H_{1} \cap K, \ldots, H_{m} \cap K\right)$ is existentially closed in

$\left(N, W_{1}, \ldots, W_{l}, H_{1}, \ldots, H_{m}\right)$ and

(b) $\left(M, V_{1}, \ldots, V_{l}, G_{1}, \ldots, G_{m}, G\right)$ is existentially closed in

$\left(K, W_{1} \cap K, \ldots, W_{l} \cap K, H_{1} \cap K, \ldots, H_{m} \cap K, H \cap K\right)$.

Proof. Since $\left(V_{1}^{+}, \ldots, V_{l}^{+}, G_{1}^{+}, \ldots, G_{m}^{+}, G^{+}\right)$is a box type, $\left(W_{i} \cap K\right)^{+}$is the unique extension of $V_{i}^{+}$for all $i \in\{1, \ldots, l\}$ and $\left(H_{i} \cap K\right)^{+}$is the unique extension of $G_{i}^{+}$on $K$ for all $i \in\{1, \ldots, m\}$.

(a). Since $\left(H_{i} \cap K\right)^{+}$is the unique extension of $G_{i}^{+}$on $K$ we get $\operatorname{sign}^{*}\left(H_{i} \cap K\right)^{+}=0$ for all $1 \leq i \leq m$ and since sign ${ }^{*} G^{+}=0$ and $\tau \models G^{+}$none of the $V\left(G_{i}\right)^{+}$is realized in $K=M\langle\tau\rangle$. It follows that all items (i)-(v) also hold true for $W_{1} \cap K, \ldots, W_{l} \cap K, H_{1} \cap K, \ldots, H_{m} \cap K$ and $W_{1}, \ldots, W_{l}, H_{1}, \ldots, H_{m}$. Hence we may use the induction hypothesis and get (a).

(b). $H^{+}$is an heir of $G^{+}$by (2.16)(iii) and our assumption (iv). Hence also $(H \cap K)^{+}$is an heir of $G^{+}$. Since $\left(V_{1}^{+}, \ldots, V_{l}^{+}, G_{1}^{+}, \ldots, G_{m}^{+}, G^{+}\right)$is a box type, item (b) of the claim holds by (6.1). This finishes the proof of claim A.

In order to prove the assertion we use (2.6):

Let $\gamma, \delta \in N, \bar{\alpha}, \bar{\beta} \in N^{l}, \bar{\gamma}, \bar{\delta} \in N^{m}$ and let $\varphi(\bar{u}, \bar{v}, x, y, \bar{x}, \bar{y})$ be an $\mathscr{L}(M)$-formula such that

$$
\begin{gathered}
N=\varphi(\bar{\alpha}, \bar{\beta}, \gamma, \delta, \bar{\gamma}, \bar{\delta}) \\
\alpha_{i}<W_{i}^{+}<\beta_{i}(1 \leq i \leq l), \\
\gamma_{j}<H_{j}^{+}<\delta_{j}(1 \leq j \leq m) \text { and } \\
\gamma<H^{+}<\delta
\end{gathered}
$$

We have to find $c, d \in M, \bar{a}, \bar{b} \in M^{l}, \bar{c}, \bar{d} \in M^{m}$ such that

$$
\begin{gathered}
M=\varphi(\bar{a}, \bar{b}, c, d, \bar{c}, \bar{d}) \\
a_{i}<V_{i}^{+}<b_{i}(1 \leq i \leq l), \\
c_{j}<G_{j}^{+}<d_{j}(1 \leq j \leq m) \text { and } \\
c<G^{+}<d .
\end{gathered}
$$

If neither $\gamma$ nor $\delta$ are realizations of $G^{+}$, then we may remove the condition $\gamma<H^{+}<\delta$ from the list and replace $\varphi$ by $\varphi \wedge x<m \wedge m^{\prime}<y$ with $m, m^{\prime} \in M, \gamma<m<G^{+}<$ $m^{\prime}<\delta$. From the induction hypothesis we get $\bar{a}, \bar{b} \in M^{l}, \bar{c}, \bar{d} \in M^{m}$ and $c, d \in M$ with

$$
\begin{gathered}
M \models \varphi(\bar{a}, \bar{b}, c, d, \bar{c}, \bar{d}) \wedge c<m \wedge m^{\prime}<d, \\
a_{i}<V_{i}^{+}<b_{i}(1 \leq i \leq l) \text { and } \\
c_{j}<G_{j}^{+}<d_{j}(1 \leq j \leq m) .
\end{gathered}
$$

These elements have the required properties for the initial problem, too.

Hence we may assume that $\gamma$ or $\delta$ realize $G^{+}$. If $\delta$ but not $\gamma$ realizes $G^{+}$, then we may remove the condition $\gamma<H^{+}$from the list and we may replace $\varphi$ by $\varphi \wedge x<m$ for some 
$m \in G, \gamma<m$. From part (a) of claim A applied to $\tau=\delta$, with $K=M\langle\delta\rangle$ we know that $\left(K, W_{1} \cap K, \ldots, W_{l} \cap K, H_{1} \cap K, \ldots, H_{m} \cap K\right)$ is existentially closed in $\left(N, W_{1}, \ldots, W_{l}\right.$, $\left.H_{1}, \ldots, H_{m}\right)$. Hence we may assume that $\bar{\alpha}, \bar{\beta} \in K^{l}, \bar{\gamma}, \bar{\delta} \in K^{m}$. Now we may use part (b) of claim A to find the required elements in $M$.

Thus we may assume that $\gamma$ is a realization of $G^{+}$. Then $\beta^{*}:=\frac{\delta}{\gamma}>V(H)^{+} \geq W_{l}^{+}$. Let $\psi(\bar{u}, \bar{v}, y, \bar{x}, \bar{y})$ be the $\mathscr{L}(M\langle\gamma\rangle)$-formula $\varphi(\bar{u}, \bar{v}, \gamma, y \cdot \gamma, \bar{x}, \bar{y})$. Then we have

$$
\begin{gathered}
N=\psi\left(\bar{\alpha}, \bar{\beta}, \beta^{*}, \bar{\gamma}, \bar{\delta}\right), \\
\alpha_{i}<W_{i}^{+}<\beta_{i}(1 \leq i \leq l), \\
\gamma_{j}<H_{j}^{+}<\delta_{j}(1 \leq j \leq m) \text { and } \\
W_{l}^{+}<\beta^{*} .
\end{gathered}
$$

Let $V_{1}^{\prime}, \ldots, V_{l}^{\prime}, G_{1}^{\prime}, \ldots, G_{m}^{\prime}$ be the convex hulls of $V_{1}, \ldots, V_{l}, G_{1}, \ldots, G_{m}$ in $M\langle\gamma\rangle$ respectively. By part (a) of claim A, there are $\beta^{\prime} \in M\langle\gamma\rangle, \bar{\alpha}^{\prime}, \bar{\beta}^{\prime} \in M\langle\gamma\rangle^{l}, \bar{\gamma}^{\prime}, \bar{\delta}^{\prime} \in M\langle\gamma\rangle^{m}$ such that

$$
\begin{gathered}
M\langle\gamma\rangle=\psi\left(\bar{\alpha}^{\prime}, \bar{\beta}^{\prime}, \beta^{\prime}, \bar{\gamma}^{\prime}, \bar{\delta}^{\prime}\right), \\
\alpha_{i}^{\prime}<V_{i}^{\prime+}<\beta_{i}^{\prime}(1 \leq i \leq l), \\
\gamma_{j}^{\prime}<G_{j}^{\prime+}<\delta_{j}^{\prime}(1 \leq j \leq m) \text { and } \\
V_{l}^{\prime+}<\beta^{\prime}
\end{gathered}
$$

If we take $\delta^{\prime}:=\gamma \cdot \beta^{\prime}$, the definition of $\psi$ says

$$
M\langle\gamma\rangle=\varphi\left(\bar{\alpha}^{\prime}, \bar{\beta}^{\prime}, \gamma, \delta^{\prime}, \bar{\gamma}^{\prime}, \bar{\delta}^{\prime}\right)
$$

Since $H^{+}$is an heir of $G^{+}$, we know from (2.4)(iv) (applied multiplicatively) that $H \cap M\langle\gamma\rangle=$ $\gamma \cdot V_{l}^{\prime}$. Consequently $V_{l}^{\prime+}<\beta^{\prime}$ implies $\gamma<(H \cap M\langle\gamma\rangle)^{+}<\gamma \cdot \beta^{\prime}=\delta^{\prime}$.

Thus we have reduced the problem, where $N=M\langle\gamma\rangle$ with some realization $\gamma$ of $G^{+}$. Now part (b) of claim A says that $\left(M, V_{1}, \ldots, V_{l}, G_{1}, \ldots, G_{m+1}\right)$ is existentially closed in $\left(N, W_{1}, \ldots, W_{l}, H_{1}, \ldots, H_{m+1}\right)$. This finishes the proof of the remarks in the case $n=0$.

The strategy of the proof of the remarks is similar to the proof just given. In order to simplify notation in the sequel, we write $G_{m+i}=V_{i}$ and $H_{m+i}=W_{i}(1 \leq i \leq l)$. We prove the assertion of the remarks by induction on $n$, where the case $n=0$ has been done above; therefore we assume the assertion for $n \geq 0$. We write $p=p_{n+1}, q=q_{n+1}$.

Claim B. If $\tau \in N$ is a realization of $p$ and $K:=M\langle\tau\rangle$, then

(a) $\left(K, H_{1} \cap K, . ., H_{m+l} \cap K, q_{1}^{L} \cap K, \ldots, q_{n}^{L} \cap K\right)$ is existentially closed in

$\left(N, H_{1}, \ldots, H_{m+l}, q_{1}^{L}, \ldots, q_{n}^{L}\right)$ and

(b) $\left(M, G_{1}, \ldots, G_{m+l}, p_{1}^{L}, \ldots, p_{n}^{L}, p^{L}\right)$ is existentially closed in

$\left(K, H_{1} \cap K, \ldots, H_{m+l} \cap K, q_{1}^{L} \cap K, \ldots, q_{n}^{L} \cap K, q^{L} \cap K\right)$.

Proof. Since $\left(G_{1}^{+}, \ldots, G_{m+l}^{+}, p_{1}, \ldots, p_{n}, p\right)$ is a box type, $q_{i} \uparrow K$ is the unique extension of $p_{i}$ on $K$ for all $1 \leq i \leq n$. Moreover, as $\operatorname{sign} p=0$, no edge of a convex subgroup of $M$ is realized in $K$.

(a). It follows that $\operatorname{sign}\left(q_{i} \uparrow K\right)=0$ for all $1 \leq i \leq n$, and items (i), (ii), (iv) and (v) also hold true for $H_{1} \cap K, \ldots, H_{m+l} \cap K, q_{1} \uparrow K, \ldots, q_{n} \uparrow K$ and $H_{1}, \ldots, H_{m+l}, q_{1}, \ldots, q_{n}$.

If $i \in\{1, \ldots, n\}$ and $f_{i}: M^{m+l} \longrightarrow M$ is an $M$-definable map with $f_{i}\left(G_{1}^{+}, \ldots, G_{m+l}^{+}\right)=\hat{p}_{i}$, then $f_{i}\left(\left(H_{1} \cap K\right)^{+}, \ldots,\left(H_{m+l} \cap K\right)^{+}\right)$is the unique extension of $\hat{p}_{i}$ on $M\langle\tau\rangle$; hence equal to $q_{i} \widehat{\uparrow}$, and item (iii) also holds true for $H_{1} \cap K, \ldots, H_{m+l} \cap K, q_{1} \uparrow K, \ldots, q_{n} \uparrow K$.

This shows that we may use the induction hypothesis to get (a).

(b). By definition (6.4), our assumption (iii) implies that $\hat{q}$ is an extension of $\hat{p}$. Since $\operatorname{sign} p=0$, we know from (2.16)(ii) that $q$ is an heir of $p$. Hence also $q \uparrow K$ is an heir of $p$. Since $\left(G_{1}^{+}, \ldots, G_{m+l}^{+}, p_{1}, \ldots, p_{n}, p\right)$ is a box type, item (b) of the claim holds by $(6.1)$. 
So we have proved claim B and we use (2.6) again for the proof of the remarks. Let $\gamma, \delta \in N$, $\bar{\alpha}, \bar{\beta} \in N^{m+l}, \bar{\gamma}, \bar{\delta} \in N^{n}$ and let $\varphi(\bar{u}, \bar{v}, x, y, \bar{x}, \bar{y})$ be an $\mathscr{L}(M)$-formula such that

$$
\begin{gathered}
N=\varphi(\bar{\alpha}, \bar{\beta}, \gamma, \delta, \bar{\gamma}, \bar{\delta}), \\
\alpha_{i}<H_{i}^{+}<\beta_{i}(1 \leq i \leq m+l), \\
\gamma_{j}<q_{j}<\delta_{j}(1 \leq j \leq n) \text { and } \\
\gamma<q<\delta .
\end{gathered}
$$

As in the proof of the case $n=0$ we may reduce to the case $\gamma \models p$ (where we use claim B instead of claim A). Then we have

$$
\beta^{*}:=\delta-\gamma>\hat{q}
$$

Let $G_{1}^{\prime}, \ldots, G_{m+l}^{\prime}$ be the convex hulls of $G_{1}, \ldots, G_{m+l}$ in $M\langle\gamma\rangle$ respectively and let $p_{1}^{\prime}, \ldots, p_{n}^{\prime}$ be the unique extensions of $p_{1}, \ldots, p_{n}$ on $M\langle\gamma\rangle$ respectively.

Let $r$ be the unique extension of $\hat{p}$ on $M\langle\gamma\rangle$.

Claim C. There are $\beta^{\prime \prime} \in M\langle\gamma\rangle, \bar{\alpha}^{\prime}, \bar{\beta}^{\prime} \in M\langle\gamma\rangle^{m+l}$ and $\bar{\gamma}^{\prime}, \bar{\delta}^{\prime} \in M\langle\gamma\rangle^{n}$ such that

$$
\begin{gathered}
M\langle\gamma\rangle=\varphi\left(\bar{\alpha}^{\prime}, \bar{\beta}^{\prime}, \gamma, \gamma+\beta^{\prime \prime}, \bar{\gamma}^{\prime}, \bar{\delta}^{\prime}\right), \\
\alpha_{i}^{\prime}<G_{i}^{\prime+}<\beta_{i}^{\prime}(1 \leq i \leq m+l), \\
\gamma_{j}^{\prime}<p_{j}^{\prime}<\delta_{j}^{\prime}(1 \leq j \leq n) \text { and } \\
r<\beta^{\prime \prime} .
\end{gathered}
$$

Suppose we have proved claim C. Since $q$ is an heir of $p$, we know that $q\lceil M\langle\gamma\rangle=\gamma+r$ is the largest extension of $p$ on $M\langle\gamma\rangle$ (cf. (2.4)(iv)). Consequently $r<\beta^{\prime \prime}$ implies $\gamma<q \mid$ $M\langle\gamma\rangle<\gamma+\beta^{\prime \prime}$. Therefore we have reduced the situation, where $N=M\langle\gamma\rangle$ with some realization $\gamma$ of $p$ and part (b) of claim B gives the assertion. It remains to prove claim $\mathrm{C}$.

Case 1: $p$ is dense, hence $r=0^{+}$. Let $\psi(\bar{u}, \bar{v}, \bar{x}, \bar{y})$ be the $\mathscr{L}(M\langle\gamma\rangle)$-formula

$$
\exists y y>0 \wedge \varphi(\bar{u}, \bar{v}, \gamma, y+\gamma, \bar{x}, \bar{y}) .
$$

Since $N=\psi(\bar{\alpha}, \bar{\beta}, \bar{\gamma}, \bar{\delta})$, part (a) of claim B and the induction hypothesis say that there are $\bar{\alpha}^{\prime}, \bar{\beta}^{\prime} \in M\langle\gamma\rangle^{m+l}$ and $\bar{\gamma}^{\prime}, \bar{\delta}^{\prime} \in M\langle\gamma\rangle^{n}$ with

$$
\begin{gathered}
M\langle\gamma\rangle \models \psi\left(\bar{\alpha}^{\prime}, \bar{\beta}^{\prime}, \bar{\gamma}^{\prime}, \bar{\delta}^{\prime}\right), \\
\alpha_{i}^{\prime}<G_{i}^{\prime+}<\beta_{i}^{\prime}(1 \leq i \leq m+l) \text { and } \\
\gamma_{j}^{\prime}<p_{j}^{\prime}<\delta_{j}^{\prime}(1 \leq j \leq n) .
\end{gathered}
$$

Now $\psi\left(\bar{\alpha}^{\prime}, \bar{\beta}^{\prime}, \bar{\gamma}^{\prime}, \bar{\delta}^{\prime}\right)$ expresses that there is some $\beta^{\prime \prime} \in M\langle\gamma\rangle$ as required in the claim.

Case 2: $p$ is not dense.

Let $f: M^{m+l} \longrightarrow M$ be an $M$-definable map with

$$
f\left(G_{1}^{+}, \ldots, G_{m+l}^{+}\right)=\hat{p} \text { and } f\left(H_{1}^{+}, \ldots, H_{m+l}^{+}\right) \leq \hat{q} .
$$

Since $\beta^{*}>\hat{q},(6.5)$ says that there are $(m+l)$-tuples $\bar{\alpha}^{*}, \bar{\beta}^{*}$, such that $\alpha_{i}^{*}<H_{i}^{+}<\beta_{i}^{*}(1 \leq$ $i \leq m+l)$ and such that $f<\beta^{*}$ on $\prod_{i=1}^{m+l}\left(\alpha_{i}^{*}, \beta_{i}^{*}\right)$. Let $\bar{u}^{* \wedge} \bar{v}^{*}$ be a new tuple of variables of length $\bar{u} \bar{v}$ and let $\psi\left(\bar{u}, \bar{v}, y, \bar{x}, \bar{y}, \bar{u}^{*}, \bar{v}^{*}\right)$ be the $\mathscr{L}(M\langle\gamma\rangle)$-formula

$$
\varphi(\bar{u}, \bar{v}, \gamma, y+\gamma, \bar{x}, \bar{y}) \wedge \forall \bar{w}\left[\bigwedge_{i=1}^{m+l} u_{i}^{*}<w_{i}<v_{i}^{*} \rightarrow f(\bar{w})<y\right] .
$$

Then we have

$$
\begin{gathered}
N=\psi\left(\bar{\alpha}, \bar{\beta}, \beta^{*}, \bar{\gamma}, \bar{\delta}, \bar{\alpha}^{*}, \bar{\beta}^{*}\right), \\
\alpha_{i}, \alpha_{i}^{*}<H_{i}^{+}<\beta_{i}, \beta_{i}^{*}(1 \leq i \leq m+l) \text { and } \\
\gamma_{j}<q_{j}<\delta_{j}(1 \leq j \leq n) .
\end{gathered}
$$


Therefore, by part (a) of claim B and the induction hypothesis, there are $\beta^{\prime \prime} \in M\langle\gamma\rangle$, $\bar{\alpha}^{\prime}, \bar{\beta}^{\prime}, \bar{\alpha}^{* *}, \bar{\beta}^{* *} \in M\langle\gamma\rangle^{m+l}, \bar{\gamma}^{\prime}, \bar{\delta}^{\prime} \in M\langle\gamma\rangle^{n}$ such that

$$
\begin{gathered}
M\langle\gamma\rangle \models \psi\left(\bar{\alpha}^{\prime}, \bar{\beta}^{\prime}, \beta^{\prime \prime}, \bar{\gamma}^{\prime}, \bar{\delta}^{\prime}, \bar{\alpha}^{* *}, \bar{\beta}^{* *}\right), \\
\alpha_{i}^{\prime}, \alpha_{i}^{* *}<G_{i}^{\prime+}<\beta_{i}^{\prime}, \beta_{i}^{* *}(1 \leq i \leq m+l) \text { and } \\
\gamma_{j}^{\prime}<p_{j}^{\prime}<\delta_{j}^{\prime}(1 \leq j \leq n) .
\end{gathered}
$$

Now $\psi$ says

$$
\begin{gathered}
M\langle\gamma\rangle \models \varphi\left(\bar{\alpha}^{\prime}, \bar{\beta}^{\prime}, \gamma, \gamma+\beta^{\prime \prime}, \bar{\gamma}^{\prime}, \bar{\delta}^{\prime}\right) \\
\quad \text { and } f\left(G_{1}^{\prime+}, \ldots, G_{m+l}^{+}\right)<\beta^{\prime \prime}
\end{gathered}
$$

But $f\left(G_{1}^{+}, \ldots, G_{m+l}^{+}\right)$is an extension of $f\left(G_{1}^{+}, \ldots, G_{m+l}^{+}\right)=\hat{p}$, hence equal to the unique extension $r$ of $\hat{p}$ on $M\langle\gamma\rangle$ and this proves claim C.

We formulate (6.6) in a special case which will be useful in applications later.

(6.8) Corollary. Let $M \prec N$ be models of $T$. Let $V_{1}, \ldots, V_{l}$ be mutually distinct, proper, convex valuation rings of $M$, and let $W_{1}, \ldots, W_{l}$ be convex valuation rings of $N$ lying over $V_{1}, \ldots, V_{l}$ respectively. Let $G_{1}, \ldots, G_{m}$ be convex subgroups of $(M,+, \leq)$ and let $H_{1}, \ldots, H_{m}$ be convex subgroups of $(N,+, \leq)$ with $G_{i}=M \cap H_{i}(1 \leq i \leq m)$. Furthermore, let $p_{1}, \ldots, p_{n}$ be cuts of $M$ and let $q_{1}, \ldots, q_{n}$ be extensions of $p_{1}, \ldots, p_{n}$ on $N$, respectively. The cases $n=0$ or $m=0$ or $l=0$ are not excluded. Suppose the following conditions hold:

(i) $\operatorname{sign} p_{i}=0(1 \leq i \leq n)$ and $\operatorname{sign}^{*} G_{i}^{+}=0(1 \leq i \leq m)$.

(ii) $\left(p_{1}, \ldots, p_{n}\right)$ and $\left(G_{1}^{+}, \ldots, G_{m}^{+}\right)$are box types.

(iii) For each $p_{i}$, which is not dense there is an $M$-definable map $f: M^{m+l} \longrightarrow M$ with $f\left(V_{1}^{+}, \ldots, V_{l}^{+}, G_{1}^{+}, \ldots, G_{m}^{+}\right)=\hat{p}_{i}$.

(iv) $H_{j}^{+}$is a coheir of $G_{j}^{+}$and $q_{k}$ is a coheir of $p_{k}(1 \leq j \leq m, 1 \leq k \leq n)$.

Then $\left(M, V_{1}, \ldots, V_{l}, G_{1}, \ldots, G_{m}, p_{1}^{L}, \ldots, p_{n}^{L}\right)$ is existentially closed in

$$
\left(N, W_{1}, \ldots, W_{l}, H_{1}, \ldots, H_{m}, q_{1}^{L}, \ldots, q_{n}^{L}\right) .
$$

Proof. We have to show conditions (iii), (iv) and (v) of (6.6). So let $p_{i}$ be not dense and let $f: M^{m+l} \longrightarrow M$ be $M$-definable with $f\left(V_{1}^{+}, \ldots, V_{l}^{+}, G_{1}^{+}, \ldots, G_{m}^{+}\right)=\hat{p}_{i}$. Since sign $p_{i}=0$ and $q_{i}$ is a coheir of $p_{i}$, we know from (2.4) that $\hat{q}_{i}$ is the largest extension of $\hat{p}_{i}$ on $N$. Consequently $f\left(W_{1}^{+}, \ldots, W_{l}^{+}, H_{1}^{+}, \ldots, H_{m}^{+}\right) \leq \hat{q}_{i}$, as follows immediately from (6.5). Hence (iii) of (6.6) holds. Similarly for (v) of (6.6): if $V\left(G_{i}\right)=V_{j}$, then $W_{j} \subseteq V\left(H_{i}\right)$, since $V\left(H_{i}\right)^{+}$ is the largest extension of $V\left(G_{i}\right)^{+}$on $N$. Finally (iv) of (6.6) holds, since $H_{i}^{+}$is an heir of $G_{i}^{+}$.

\section{Model COMPleteness OF EXHAUSTIVE EXPANSIONS}

By applying Theorem (6.6) and the Robinson test we are looking for model completeness results for certain convex expansions of a polynomially bounded structure $M$. These expansions should be

(a) exhaustive, in the sense that every expansion of $M$ by finitely many convex subsets of $M$ is interdefinable (with parameters) with one such expansion, and

(b) as simple as possible in terms of new symbols that are needed in order to obtain model completeness.

In particular we only want to introduce names for subsets of $M$ if possible. This concerns mainly item (iii) in (6.6) and we will narrow the class of functions needed in this condition. 
This is done via a generalization the following consequence of the valuation property (cf. [vdD-Sp], 9.2).

(7.1) Proposition. ([Tr2], (4.2))

Let $G \subseteq M \models T$ be a convex subgroup and let $f: M \longrightarrow M$ be $M$-definable. Then there are an exponent $\lambda$ and $a, b \in M$ with $f\left(G^{+}\right)=a+b \cdot\left(G^{+}\right)^{\lambda}$.

(7.2) Proposition. Let $G_{1}, \ldots, G_{n}, H_{1}, \ldots, H_{k}$ be convex subgroups of $(M,+, \leq)$ such that $\left(G_{1}^{+}, \ldots, G_{n}^{+}, H_{1}^{+}, \ldots, H_{k}^{+}\right)$is a box type and let $p$ be a cut of $M$ with $V(p) \neq V\left(H_{1}\right), \ldots, V\left(H_{k}\right)$. If there is an $M$-definable map $f: M^{n} \times M^{k} \longrightarrow M$ with $p=f\left(G_{1}^{+}, \ldots, G_{n}^{+}, H_{1}^{+}, \ldots, H_{k}^{+}\right)$, then there are $a, b \in M$ and exponents $\lambda_{1}, \ldots, \lambda_{n}$ such that

$$
p=a+b \cdot\left(G_{1}^{+}\right)^{\lambda_{1}} \cdot \ldots \cdot\left(G_{n}^{+}\right)^{\lambda_{n}} .
$$

ProOF. The expression $a+b\left(G_{1}^{+}\right)^{\lambda_{1}} \ldots\left(G_{n}^{+}\right)^{\lambda_{n}}$ here denotes the result of applying the function $a+b \cdot x_{1}^{\lambda_{1}} \cdot \ldots \cdot x_{n}^{\lambda_{n}}$ to the box type $\left(G_{1}^{+}, \ldots, G_{n}^{+}\right)$. Observe that $\left(G_{1}^{+}\right)^{\lambda_{1}} \cdot \ldots \cdot\left(G_{n}^{+}\right)^{\lambda_{n}}$ is the upper edge of a convex subgroup of $(M,+, \leq)$ if at least one of the $\lambda_{i}$ is non zero; the set of positive elements of this group is $\left\{a_{1}^{\lambda_{1}} \cdot \ldots \cdot a_{n}^{\lambda_{n}} \mid a_{1}, \ldots, a_{n}>0, \lambda_{i} \geq 0 \Rightarrow a_{i} \in G_{i}, \lambda_{i}<0 \Rightarrow a_{i}>G_{i}\right\}$.

For the proof we may reindex the $G_{i}, H_{j}$ if necessary such that $V\left(G_{i}\right)=V(p)$ for all $i \in\{1, \ldots, n\}$. We write $V$ for this valuation ring. By (5.3), we know that there is an $M$ definable map $g: M^{n} \longrightarrow M$ with $p=g\left(G_{1}^{+}, \ldots, G_{n}^{+}\right)$. By $(5.4), \operatorname{sign} p \neq 0$ and $p=a+b \cdot U^{+}$ for some $a, b \in M$ and $U=G(p)$. We prove the proposition by induction on $n$, starting from $n=1$, where we may use (7.1). Since $\operatorname{sign} p \neq 0$ we may assume that $p=U^{+}$with $U=G(p)$. Since $V=V\left(G_{1}\right)=\ldots=V\left(G_{n}\right)$ and $\left(G_{1}^{+}, \ldots, G_{n}^{+}\right)$is a box type, there is at most one $i$ with $\operatorname{sign}^{*} G_{i}^{+} \neq 0$ and we may assume that $\operatorname{sign}^{*} G_{n}^{+}=0$. By the induction hypothesis we may also assume that $\left(G_{1}^{+}, \ldots, G_{n-1}^{+}, U^{+}\right)$is a box type and that $U^{+}$is omitted in $N$.

Let $\alpha$ be a realization of $G_{n}^{+}$and let $N:=M\langle\alpha\rangle$. Let $G_{i}^{\prime}$ and $U^{\prime}$ be the convex hulls of $G_{i}$, $U$, respectively in $N(1 \leq i<n)$. By $(3.4),\left(G_{1}^{\prime+}, \ldots, G_{n-1}^{++}\right)$is a box type. Since $U^{+}$and $G_{i}^{+}$ are omitted in $N$ for all $1 \leq i<n, g\left(G_{1}^{+}, \ldots, G_{n}^{+}\right)=U^{+}$implies that $g\left(G_{1}^{\prime+}, \ldots, G_{n-1}^{\prime+}, \alpha\right)=$ $U^{\prime+}$ and by the induction hypothesis there are exponents $\lambda_{1}, \ldots, \lambda_{n-1}$, not all equal to 0 and $\delta, \gamma \in N$ with $U^{\prime+}=\delta+\gamma \cdot\left(G_{1}^{\prime+}\right)^{\lambda_{1}} \cdot \ldots \cdot\left(G_{n-1}^{\prime+}\right)^{\lambda_{n-1}}$. Since $U^{\prime}$ is a convex subgroup of $(N,+, \leq)$ we may assume that $\delta=0$. If $\gamma \in M$, then we are done. Hence we may assume that $\gamma$ realizes a cut $q$ of $M$ and from the case $n=1$ we get $a, b \in M$ and an exponent $\lambda_{n} \neq 0$ such that $q=a+b \cdot\left(G_{n}^{+}\right)^{\lambda_{n}}$. It follows

$$
U^{+}=\left[a+b \cdot\left(G_{n}^{+}\right)^{\lambda_{n}}\right] \cdot\left(G_{1}^{+}\right)^{\lambda_{1}} \cdot \ldots \cdot\left(G_{n-1}^{+}\right)^{\lambda_{n-1}} .
$$

If $a=0$ we are done. Hence we may assume that $a \neq 0$. Let $G$ be the convex subgroup of $(M,+, \leq)$ with $G^{+}=\left(G_{1}^{+}\right)^{\lambda_{1}} \ldots \cdot\left(G_{n-1}^{+}\right)^{\lambda_{n-1}}$. Then $G_{n}^{+} \not G^{+}$and by $(5.1), V(G)=V$ again. Let $\beta$ be a realization of $G^{+}$. In order to prove the proposition we show that $\left(a+b \alpha^{\lambda_{n}}\right) \cdot \beta$ realizes $a \cdot G^{+}$or $b \cdot\left(G_{n}^{+}\right)^{\lambda_{n}} \cdot G^{+}$- then by $(*), U^{+}=a \cdot G^{+}$or $U^{+}=b \cdot\left(G_{n}^{+}\right)^{\lambda_{n}} \cdot G^{+}$, which gives the assertion.

Claim 1. If there is some $c \in M$ with $|a| \cdot G^{+}<c<|b| \cdot\left(G_{n}^{+}\right)^{\lambda_{n}} \cdot G^{+}$, then $\left(a+b \alpha^{\lambda_{n}}\right) \cdot \beta$ realizes the same cut of $M$ as $b \alpha^{\lambda_{n}} \beta$.

Proof. Since $b \alpha^{\lambda_{n}} \beta$ realizes the edge of a convex subgroup of $(M,+, \leq), c$ is in this group. Thus $b \alpha^{\lambda_{n}} \beta-c$ and $b \alpha^{\lambda_{n}} \beta+c$ also realize this edge. Since $|a| \cdot G^{+}<c$ we have $b \alpha^{\lambda_{n}} \beta-c<\left(a+b \alpha^{\lambda_{n}}\right) \cdot \beta<b \alpha^{\lambda_{n}} \beta+c$, which implies claim 1.

Claim 2. If there is some $c \in M$ with $|a| \cdot G^{+}>c>|b| \cdot\left(G_{n}^{+}\right)^{\lambda_{n}} \cdot G^{+}$, then $\left(a+b \alpha^{\lambda_{n}}\right) \cdot \beta$ realizes the same cut of $M$ as $a \beta$. 
Proof. As in the proof of claim 1 with interchanged roles of $a \beta$ and $b \alpha^{\lambda_{n}} \beta$.

By claim 1 and 2 it remains to show

Claim 3. $|a| \cdot G^{+} \neq|b| \cdot\left(G_{n}^{+}\right)^{\lambda_{n}} \cdot G^{+}$.

Proof. Since sign* $G_{n}^{+}=0$ and $\alpha \models G_{n}^{+}$, the upper edge of $V=V\left(G_{n}\right)$ is omitted in $N=M\langle\alpha\rangle$. Let $W$ be the convex hull of $V$ in $N$. Suppose $|a| \cdot G^{+}=|b| \cdot\left(G_{n}^{+}\right)^{\lambda_{n}} \cdot G^{+}$. Then $|a| \cdot \beta$ and $|b| \cdot \alpha^{\lambda_{n}} \cdot \beta$ realize the same cut of $M$, namely $|a| \cdot G^{+}$. Since $G_{n}^{+} \not G^{+}$, the upper edge of the convex hull $G^{\prime}$ of $G$ in $N$ is the unique extension of $G^{+}$on $N$; hence $V\left(G^{\prime}\right)$ lies over $V(G)=V$ and $V\left(G^{\prime}\right)=W$. Moreover, $|a| \cdot \beta$ and $|b| \cdot \alpha^{\lambda_{n}} \cdot \beta$ realize $|a| \cdot G^{\prime+}$, hence $\alpha^{\lambda_{n}} \frac{|b|}{|a|} \in V\left(G^{\prime}\right)^{*}=W^{*}$ and there are $c, d \in V^{*}$, with $0<c<\alpha^{\lambda_{n}} \frac{|b|}{|a|}<d$. But this means that $\frac{d}{c}$ is not in the multiplicative invariance group of $\frac{|b|}{|a|} \cdot\left(G_{n}^{+}\right)^{\lambda_{n}}$, in contradiction to $\frac{d}{c} \in V^{*>0}$ and $V=V\left(G_{n}\right)=V\left(\frac{|b|}{|a|} \cdot\left(G_{n}^{+}\right)^{\lambda_{n}}\right)$. This proves claim 3 and the proposition.

(7.3) Definition. An exhaustive extension of $T$ is a theory $T^{*}$ in a language $\mathscr{L}^{*}$ such that

(i) $\mathscr{L}^{*}$ is the language $\mathscr{L}$ together with new unary predicates

$$
\Theta_{1}, \ldots, \Theta_{l}, \mathcal{G}_{1}, \ldots, \mathcal{G}_{m}, \mathcal{D}_{1}, \ldots, \mathcal{D}_{n} \text { for some } l, m, n \geq 0
$$

(ii) $T^{*}$ is an $\mathscr{L}^{*}$-theory extending $T$ which is formed in the following way. There is a partition $N_{1} \cup N_{2}$ of $\{1, \ldots, n\} \quad\left(N_{1}\right.$ or $N_{2}$ may be empty), for each $i \in N_{1}$ an $(l+m)$ tuple $\lambda_{i}=\left(\lambda_{i}(1), \ldots, \lambda_{i}(l+m)\right)$ of exponents, and a map $\sigma:\{1, \ldots, m\} \longrightarrow\{1, \ldots, l\}$, such that $T^{*}$ says the following about a model $\left(M, V_{1}, \ldots, V_{l}, G_{1}, \ldots, G_{m}, D_{1}, \ldots, D_{n}\right)$ :

1. $V_{1}, \ldots, V_{l}$ are mutually distinct, proper, convex valuation rings of $M, G_{1}, \ldots, G_{m}$ are convex subgroups of $(M,+, \leq)$ and $D_{1}, \ldots, D_{n}$ are left options of cuts of $M$.

2. $\operatorname{sign} D_{i}^{+}=0(1 \leq i \leq n)$ and $\operatorname{sign}^{*} G_{i}^{+}=0(1 \leq i \leq m)$.

3. $\left(D_{1}^{+}, \ldots, D_{n}^{+}\right)$and $\left(G_{1}^{+}, \ldots, G_{m}^{+}\right)$are box types.

4. $D_{i}^{+}$is a dense cut $\left(i \in N_{2}\right)$.

5. For each $i \in N_{1}, D_{i}^{+}$is not dense and

$$
\left(V_{1}^{+}\right)^{\lambda_{i}(1)} \cdot \ldots \cdot\left(V_{l}^{+}\right)^{\lambda_{i}(l)} \cdot\left(G_{1}^{+}\right)^{\lambda_{i}(l+1)} \cdot \ldots \cdot\left(G_{m}^{+}\right)^{\lambda_{i}(l+m)}=\widehat{D_{i}^{+}} .
$$

6. For all $i \in\{1, \ldots, m\}$, we have $V_{\sigma i}=V\left(G_{i}\right)$.

(iii) $T^{*}$ is consistent.

The structure $\left(M, V_{1}, \ldots, V_{l}, G_{1}, \ldots, G_{m}, p_{1}^{L}, \ldots, p_{n}^{L}\right)$ is called an exhaustive expansion of $M$. We shall also say that $\left(M, V_{1}^{+}, \ldots, V_{l}^{+}, G_{1}^{+}, \ldots, G_{m}^{+}, p_{1}, \ldots, p_{n}\right)$ is an exhaustive expansion of $M$.

Observe that indeed 1.-6. are axiomatizable in $\mathscr{L}^{*}$. According to $(3.2)(\mathrm{i}) \Leftrightarrow($ ii), the property of a tuple $\left(p_{1}, \ldots, p_{n}\right)$ of cuts being a box type in condition 3 can be formulated by the infinite scheme of sentences which says that for every $k<n$ and for every definable function $f$ in $k$ variables, with parameters, there is an open box $B$ around $\left(p_{1}, \ldots, p_{k}\right)$ and some $z$ such that $\left.f\right|_{B} \leq z<p_{k+1}$ or $p_{k+1}<z \leq\left. f\right|_{B}$.

(7.4) TheOREm. If $T$ is model complete then each exhaustive extension of $T$ is model complete, too.

Proof. Let $T^{*}$ denote the theory in question and let

$$
M^{*}:=\left(M, V_{1}, \ldots, V_{l}, G_{1}, \ldots, G_{m}, p_{1}, \ldots, p_{n}\right)
$$




$$
N^{*}:=\left(N, W_{1}, \ldots, W_{l}, H_{1}, \ldots, H_{m}, q_{1}, \ldots, q_{n}\right)
$$

be models of $T^{*}$ with $M^{*} \subseteq N^{*}$. Then properties (i)-(iii) of (6.6) are fulfilled for the extension $M^{*} \subseteq N^{*}$. Since $\bar{V}\left(G_{i}\right)=V_{\sigma i}$ and $V\left(H_{i}\right)=W_{\sigma i}$ by the axioms of $T^{*}$, we also know (iv) and (v) of (6.6). By (6.6), $M^{*}$ is existentially closed in $N^{*}$. Hence Robinson's test gives us model completeness of $T^{*}$.

(7.5) Corollary. Let $\mathscr{M}=\left(M, q_{1}, \ldots, q_{k}\right)$ be exhaustive and let $h \in\{1, \ldots, k\}$.

(i) $\left(M, q_{1}, \ldots, q_{h}\right)$ is exhaustive, too.

(ii) Let $N:=M\left\langle\alpha_{h+1}, \ldots, \alpha_{k}\right\rangle$ with realizations $\alpha_{i}$ of $q_{i}(h<i \leq k)$. Then each $q_{i}$ with $i \leq h$ has a unique extension $r_{i}$ on $N,\left(N, r_{1}, \ldots, r_{h}\right)$ is exhaustive and an elementary extension of $\left(M, q_{1}, \ldots, q_{h}\right)$.

Proof. (i) follows directly from definition (7.3).

(ii). By (5.4), $\left(q_{1}, \ldots, q_{k}\right)$ is a box type, hence each $q_{i}$ with $i \leq h$ has a unique extension $r_{i}$ on $N$ and by $(2.4), r_{i}$ has the same signature and the same multiplicative signature as $q_{i}$. Moreover, by $(3.4),\left(r_{1}, \ldots, r_{h}\right)$ is a box type, too. This shows that 1-3 of (7.3) also hold true for $\left(N, r_{1}, \ldots, r_{h}\right)$ and the partition of $r_{1}, \ldots, r_{h}$ into upper edges of valuation rings, groups and cuts is inherited from $\left(M, q_{1}, \ldots, q_{h}\right)$. In order to prove (ii) we may use (7.4) and it is enough to show that conditions $4-6$ of (7.3) inherited from $\left(M, q_{1}, \ldots, q_{h}\right)$ also hold for $\left(N, r_{1}, \ldots, r_{h}\right)$.

Condition 6 holds for $\left(N, r_{1}, \ldots, r_{h}\right)$ and the map $\sigma$ inherited from $\left(M, q_{1}, \ldots, q_{h}\right)$, since in the case $r_{i}=\hat{r}_{i}, \operatorname{sign}^{*} r_{i}=0$ we have $q_{i}=\hat{q}_{i}, \operatorname{sign}^{*} q_{i}=0$, hence $V\left(q_{i}\right)^{+}$is among the $q_{1}, \ldots, q_{i-1}$ and therefore omitted in $N$.

Condition 4 holds for $\left(N, r_{1}, \ldots, r_{h}\right)$, since $r_{i}$ is dense if and only if $q_{i}$ is dense: this follows from (2.4), since $M$ is archimedean in $N$ and $q_{i}$ is omitted in $N$.

Condition 5 of $(7.3)$ holds for $\left(N, r_{1}, \ldots, r_{h}\right)$ and the same vectors of exponents valid for $\left(M, q_{1}, \ldots, q_{h}\right)$ : Let $i \in\{1, \ldots, h\}$ such that $\operatorname{sign} q_{i}=0$. By definition (7.3), also sign $q_{i}=$ $\ldots=\operatorname{sign} q_{k}=0$. Then by (5.4), no cut of $M$ with signature $\neq 0$ is realized in $N$. Hence if $\lambda$ is a vector of exponents of length $\rho$ such that $q_{1}^{\lambda(1)} \cdot \ldots \cdot q_{\rho}^{\lambda(\rho)}=\hat{q}_{i}$, then $r_{1}^{\lambda(1)} \cdot \ldots \cdot r_{\rho}^{\lambda(\rho)}$ is the unique extension of $\hat{q}_{i}$ on $N$, which is equal to $\hat{r}_{i}$ as $r_{i}$ is the unique extension of $q_{i}$ on $N$.

Next we show that every expansion of a model $M$ of $T$ by convex subsets of $M$ is interdefinable, using parameters, with some exhaustive expansion of $M$.

(7.6) Proposition. Let $M$ be a model of $T$ and let $q_{1}, \ldots, q_{k}$ be nonprincipal cuts of $M$. Then there is an exhaustive extension $T^{*}$ of $T h(M)$ and an expansion $M^{*}$ of $M$, such that $M^{*}=T^{*}$ and such that $M^{*}$ and $\left(M, q_{1}^{L}, \ldots, q_{k}^{L}\right)$ are interdefinable with parameters from $M$. The expansion $M^{*}$ is formed in the following way:

Let $\left(V_{1}, \ldots, V_{l}\right)$ be an enumeration of $\left\{V\left(q_{i}\right) \mid V\left(q_{i}\right) \neq M\right\}$. Let $\left(G_{1}^{+}, \ldots, G_{m}^{+}\right)$be an enumeration of a maximal box type in $\left\{\hat{q}_{i} \mid \operatorname{sign}^{*} \hat{q}_{i}=0\right\}$ and let $\left(r_{1}, \ldots, r_{n}\right)$ be an enumeration of a maximal box type in $\left\{q_{i} \mid \operatorname{sign} q_{i}=0\right\}$. Let

$I:=\left\{i \in\{1, \ldots, n\} \mid \operatorname{sign}^{*} \hat{r}_{i}=0\right\}$ and $J:=\left\{i \in\{1, \ldots, n\} \mid r_{i}\right.$ not dense and $\left.\operatorname{sign}^{*} \hat{r}_{i} \neq 0\right\}$.

Then for each $i \in I$ there are $b_{i} \in M, b_{i}>0$ and an $m$-tuple $\mu_{i}=\left(\mu_{i}(1), \ldots, \mu_{i}(m)\right)$ of exponents with $\left(G_{1}^{+}\right)^{\mu_{i}(1)} \cdot \ldots \cdot\left(G_{m}^{+}\right)^{\mu_{i}(m)}=b_{i} \cdot \hat{r}_{i}$ and for each $i \in J$ there are $b_{i} \in M, b_{i}>0$ and $\varepsilon_{i} \in\{ \pm 1\}$ such that $b_{i} \cdot \hat{r}_{i}=\left(V\left(r_{i}\right)^{+}\right)^{\varepsilon_{i}}$. Define for $i \in\{1, \ldots, n\}: p_{i}:=b_{i} \cdot r_{i}$.

Then we take $M^{*}=\left(M, V_{1}, \ldots, V_{l}, G_{1}, \ldots, G_{m}, p_{1}, \ldots, p_{n}\right)$ and $T^{*}=T h\left(M^{*}\right)$.

Proof. If $i \in I$, then by choice of the $G_{j}$ there is an $M$-definable map $f$ with $\hat{r}_{i}=$ $f\left(G_{1}^{+}, \ldots, G_{m}^{+}\right)$. Hence the existence of $\mu_{i}$ and $b_{i}$ is justified by (7.2). We have to show 
that $M^{*}$ is an exhaustive expansion of $M$. Let $N_{1}=\left\{i \in\{1, . ., n\} \mid p_{i}\right.$ is not dense $\}$, $N_{2}=\{1, \ldots, n\} \backslash N_{1}$ and let $\sigma:\{1, \ldots, m\} \longrightarrow\{1, \ldots, l\}$ be defined by $V_{\sigma i}=V\left(G_{i}\right)$. For each $i \in N_{1}$ we define an $(l+m)$-tuple $\lambda_{i}=\left(\lambda_{i}(1), \ldots, \lambda_{i}(l+m)\right)$ of exponents as follows:

- If $i \in I$, then we take $\left(\lambda_{i}(1), \ldots, \lambda_{i}(l+m)\right)=\left(0, \ldots, 0, \mu_{i}(1), \ldots, \mu_{i}(m)\right)$.

- If $i \in J$, then we take $\lambda_{i}(j)=\varepsilon_{i}$ for the index $j \in\{1, \ldots, l\}$ with $V\left(r_{i}\right)=V_{j}$ and $\lambda_{i}(s)=0$ for each other $s \in\{1, \ldots, l+m\}$.

Since $b_{i} \cdot \hat{r}_{i}=\widehat{b_{i} \cdot r_{i}}=\hat{p}_{i}$, the choice of the $b_{i}$ implies that all conditions of definition (7.3) are satisfied for $M^{*}$. Hence $M^{*}$ is exhaustive and the definition of $T^{*}$ (and $\mathscr{L}^{*}$ ) is the natural one, which turns $M^{*}$ into a model of $T^{*}$.

It is straightforward to check that each $q_{i}^{L}$ is definable in $M^{*}$ with parameters from $M$, hence $M^{*}$ has the required properties.

(7.7) Corollary. Let $T$ be model complete and let $M \models T$.

(a) Let $\mathbb{V}$ be the set of all cuts $V^{+}$, where $V$ is a proper convex valuation ring $V$ of $M$.

(b) Let $\mathbb{G}$ be a set of cuts of the form $G^{+}$, where $G$ is a convex subgroup of $(M,+, \leq)$ with sign $^{*} G^{+}=0$, such that $\mathbb{G}$ is maximal with the property that any finite sequence in $\mathbb{G}$ is a box type.

(c) Let $\mathbb{E}_{0}$ be a set of cuts of signature 0 , such that $\mathbb{E}_{0}$ is maximal with the property that any finite sequence in $\mathbb{E}_{0}$ is a box type.

(d) For each $p \in \mathbb{E}_{0}$ there is a finite tuple $\bar{q}$ of cuts from $\mathbb{V} \cup \mathbb{G}$ and some $b_{p} \in M$ so that $b_{p} \cdot \hat{p}=f(\bar{q})$ with some function $f(\bar{x})=\prod x_{i}^{\lambda_{i}}$ and some exponents $\lambda_{i}$.

Let $\mathbb{E}:=\left\{b_{p} \cdot p \mid p \in \mathbb{E}_{0}\right\}$.

Let $\mathbb{D}:=\mathbb{V} \cup \mathbb{G} \cup \mathbb{E}$. Then

(i) $\left(M,\left(p^{L} \mid p \in \mathbb{D}\right)\right)$ is model complete in the language which extends $\mathscr{L}$ and has a unary predicate for every $p^{L}(p \in \mathbb{D})$, and

(ii) every convex subset of $M$ is definable in $\left(M,\left(p^{L} \mid p \in \mathbb{D}\right)\right)$ with parameters.

Proof. By (7.6).

(7.8) REMARK. I do not know if the structure $\mathscr{M}:=\left(M,\left(p^{L} \mid p\right.\right.$ a nonprincipal cut of $\left.\left.M\right)\right)$ is model complete in general and I conjecture that it is not. Clearly (7.7) implies that $\mathscr{M}$ is model complete if we add a constant symbol for each element of $M$.

\section{StruCture theOREMS FOR EXHAUSTIVE EXPANSIONS}

(8.1) Theorem. Let $\mathscr{M}:=\left(M, V_{1}, \ldots, V_{l}, G_{1}, \ldots, G_{m}, p_{1}^{L}, \ldots, p_{n}^{L}\right)$ be an exhaustive expansion of some $T$-model $M$ in the language $\mathscr{L}^{*}$. Let $\alpha_{i} \models V_{i}^{+}, \beta_{i} \models G_{i}^{+}, \gamma_{i} \models p_{i}$ and let

$$
M^{\prime}:=M\langle\bar{\beta}, \bar{\gamma}\rangle .
$$

Let $H_{j}$ be convex subgroups of $M^{\prime}$, such that $H_{j}^{+}$is a coheir of $G_{j}^{+}$and let $q_{i}$ be a coheir of $p_{i}$ on $M^{\prime}(1 \leq j \leq m, 1 \leq i \leq n)$. Let $W_{i}$ be the convex hull of $V_{i}$ in $M^{\prime}$ and let

$$
\mathscr{M}^{\prime}:=\left(M^{\prime}, W_{1}, \ldots, W_{l}, H_{1}, \ldots, H_{m}, q_{1}^{L}, \ldots, q_{n}^{L}\right) .
$$

Then for each $r \in \mathbb{N}$ and all sets $X \subseteq M^{r}$, 0-definable in $\mathscr{M}$ we have: 
(i) If $X$ is defined by the $\mathscr{L}^{*}$-formula $\varphi(\bar{x})=\exists \bar{u}[\vartheta(\bar{u}, \bar{x}) \wedge \chi(\bar{u}, \bar{x})]$, where $\chi$ is an $\mathscr{L}$-formula and $\vartheta$ is a quantifier free $\mathscr{L}^{*}$-formula, then the set $X^{\prime} \subseteq M^{\prime r}$ defined by $\varphi(\bar{x})$ in $\mathscr{M}^{\prime}$ has the property $X^{\prime} \cap M^{r}=X$.

(ii) With $N:=M^{\prime}\langle\bar{\alpha}\rangle=M\langle\bar{\alpha}, \bar{\beta}, \bar{\gamma}\rangle$, there is an $\{\bar{\alpha}, \bar{\beta}, \bar{\gamma}\}$-definable set $Y \subseteq N^{r}$ such that $X=Y \cap M^{r}$.

Proof. We may assume that $T=T^{d f}$ has quantifier elimination and a universal system of axioms in the language $\mathscr{L}$. Then $\varphi(\bar{x})$ may be replaced by an existential $\mathscr{L}^{*}$-formula.

(i). By $(6.8), \mathscr{M}$ is existentially closed in $\mathscr{M}^{\prime}$. Since $\varphi(\bar{x})$ is an existential formula, this implies $X^{\prime} \cap M^{k}=X$.

(ii). By (7.4), $X$ is defined by an existential $\mathscr{L}^{*}$-formula $\varphi(\bar{x})$. Take $X^{\prime}$ as in (i). By (2.4)(iv), we have $\operatorname{sign}^{*} H_{j}^{+} \neq 0$ and $\operatorname{sign} q_{i} \neq 0$. Thus $H_{1}, \ldots, H_{m}$ and $q_{1}^{L}, \ldots, q_{n}^{L}$ are definable in $\left(M^{\prime}, W_{1}, \ldots, W_{l}\right)$ with parameters from $\{\bar{\beta}, \bar{\gamma}\}$. By $(6.3), T h\left(M^{\prime}, W_{1}, \ldots, W_{l}\right)$ has quantifier elimination in the language $\mathscr{L}\left(\Theta_{1}, \ldots, \Theta_{l}\right)$, hence $X^{\prime}$ is a boolean combination of sets of the form

$$
Y^{\prime}:=\left\{\bar{b} \in M^{\prime r} \mid \psi(\bar{b}) \wedge f(\bar{b}) \in O_{i}\right\}
$$

where $\psi(\bar{x})$ is a quantifier free $\mathscr{L}$-formula with parameters from $\{\bar{\beta}, \bar{\gamma}\}$ and $f: M^{\prime r} \longrightarrow M^{\prime}$ is $\{\bar{\beta}, \bar{\gamma}\}$-definable. Since $\alpha_{i}$ is a realization of $W_{i}^{+}$we get $Y^{\prime}=Y \cap M^{\prime r}$, where

$$
Y:=\left\{\bar{\beta} \in N^{r}|\psi(\bar{\beta}) \wedge| f(\bar{\beta}) \mid<\alpha_{i}\right\} .
$$

This shows that $X^{\prime}$ is a boolean combination of traces of $\{\bar{\alpha}, \bar{\beta}, \bar{\gamma}\}$-definable subsets $Y$ of $N^{r}$. Consequently, also $X$ is a boolean combination of traces of $\{\bar{\alpha}, \bar{\beta}, \bar{\gamma}\}$-definable subsets $Y$ of $N^{r}$. Since taking traces commutes with all boolean operations we get the assertion.

(8.2) Corollary. Let $M \models T$ and let $D_{1}, \ldots, D_{n}$ be left options of cuts of $M$. Then there is an elementary extension $N$ of $M$ with $\operatorname{dim}_{M} N \leq 3 n$, such that for all $k \in \mathbb{N}$ and each $X \subseteq M^{k}$, definable in $\left(M, D_{1}, \ldots, D_{n}\right)$ with parameters from $M$, there is an $N$-definable set $Y \subseteq N^{k}$ with $X=Y \cap M^{k}$.

Proof. By (7.6), the sets $D_{i}$ are definable in some exhaustive expansion

$$
\left(M, V_{1}, \ldots, V_{l}, G_{1}, \ldots, G_{m}, p_{1}^{L}, \ldots, p_{n^{\prime}}^{L}\right)
$$

of $M$, with some $m, l, n^{\prime} \leq n$. Now we may apply (8.1).

(8.3) Remark. The bound $3 n$ in (8.2) is sharp. To see this, take convex valuation rings $V_{1} \varsubsetneqq \ldots \varsubsetneqq V_{n} \varsubsetneqq M$ of a $T$-model $M$ and cuts $p_{1}, \ldots, p_{n}$ of $M$ with sign $p_{i}=\operatorname{sign}^{*} \hat{p}_{i}=0$ and $V\left(p_{i}\right)=V_{i}(1 \leq i \leq n)$. This situation can be produced by using (2.15). By (4.2), each $\left(p_{i}, \hat{p}_{i}, V\left(p_{i}\right)^{+}\right)$is a box type. By $(5.1),\left(V\left(p_{1}\right)^{+}, \ldots, V\left(p_{n}\right)^{+}, \hat{p}_{1}, \ldots, \hat{p}_{n}, p_{1}, \ldots, p_{n}\right)$ is a box type, too. Hence if $N$ is an elementary extension of $M$ of dimension $<3 n$ over $M$, then the set $V\left(p_{1}\right) \times \ldots \times V\left(p_{n}\right) \times G\left(p_{1}\right) \times \ldots \times G\left(p_{n}\right) \times p_{1}^{L} \times \ldots \times p_{n}^{L}$ can not be the trace of an $N$-definable subset of $N^{3 n}$.

(8.4) Proposition. Let $\Theta_{1}, \ldots, O_{l}$ be new unary predicates and let $c_{1}, \ldots, c_{l}$ be new constants. Let $\mathscr{L}_{\text {convex }, l}:=\mathscr{L}\left(\Theta_{1}, \ldots, \Theta_{l}\right)$ and let $T_{\text {convex }, l}$ be the $\mathscr{L}_{\text {convex }, l}$-theory as in (6.3). Let $T^{*}$ be the $\mathscr{L}_{\text {convex }, l}(\bar{c})$-theory which extends $T_{\text {convex }, l}$ and which says in addition that $\Theta_{1}<c_{1} \in \Theta_{2}<c_{2} \in \ldots \in \Theta_{l}<c_{l}$. If $\varphi(\bar{x}, \bar{y})$ is an $\mathscr{L}_{\text {convex }, l}(\bar{c})$-formula, then there are $\mathscr{L}_{\text {convex }, l}$-formulas $\psi_{1}(\bar{x}, \bar{u}), \ldots, \psi_{m}(\bar{x}, \bar{u})$ and $\bar{y}$-tuples of 0 -definable $\mathscr{L}$-functions 
$\bar{f}_{1}(\bar{x}, \bar{u}), \ldots, \bar{f}_{m}(\bar{x}, \bar{u})$, such that

$$
T^{*} \vdash\left[\forall \bar{x} \exists ! i \in\{1, \ldots, m\} \psi_{i}(\bar{x}, \bar{c})\right] \wedge \bigwedge_{i=1}^{m}\left[\forall \bar{x} \psi_{i}(\bar{x}, \bar{c}) \rightarrow \varphi\left(\bar{x}, \bar{f}_{i}(\bar{x}, \bar{c})\right)\right] .
$$

Hence " $T^{*}$ has definable Skolem functions, which are $\bar{c}$-definable in $T$ on finitely many $T^{*}$-definable pieces".

Proof. We may assume that $T=T^{d f}$ is model complete and universally axiomatized. Then $T^{*}$ is model complete and axiomatized by a set of universal $\mathscr{L}^{*}$-axioms. In this situation we may apply Herbrand's Theorem (cf. [Sho]) which implies that every universal and model complete theory in a language has definable Skolem functions, which are given by terms in that language on finitely many definable pieces.

(8.5) Theorem. (Functions, definable in exhaustive expansions)

Let $\mathscr{M}$ be an exhaustive expansion of a T-model $M$ in the language $\mathscr{L}^{*}$. If $f: M^{n} \longrightarrow M^{k}$ is 0 -definable in $\mathscr{M}$, then there are $\mathscr{L}^{*}$-formulas $\psi_{1}(\bar{x}), \ldots, \psi_{m}(\bar{x})$ and 0 -definable $\mathscr{L}$-functions $g_{1}(\bar{x}), \ldots, g_{m}(\bar{x})$, such that

$$
T^{*} \vdash\left[\forall \bar{x} \exists ! i \in\{1, \ldots, m\} \psi_{i}(\bar{x})\right] \wedge \bigwedge_{i=1}^{m}\left[\forall \bar{x} \psi_{i}(\bar{x}) \rightarrow f(\bar{x})=g_{i}(\bar{x})\right] .
$$

Proof. Let $\mathscr{M}=\left(M, V_{1}, \ldots, V_{l}, p_{1}^{L}, \ldots, p_{r}^{L}\right)$ with convex valuation rings $V_{i}$ and cuts $p_{i}$ with $\operatorname{sign} p_{i}=0$ or $p_{i}=\hat{p}_{i}$ and $\operatorname{sign}^{*} \hat{p}_{i}=0$. We prove the assertion by induction on $r$. Again we may assume that $T=T^{d f}$ and $\mathscr{L}=\mathscr{L}^{d f}$.

$\underline{r=0}$. Thus $\mathscr{M}=\left(M, V_{1}, \ldots, V_{l}\right) \models T_{\text {convex }, l}$, which is a complete theory. Firstly for every model $\mathscr{N}$ of $T_{\text {convex }, l}$ and every $A \subseteq \mathscr{N}$, the definable closure of $A$ in $\mathscr{N}$ is the definable closure $P$ of $A$ in $N:=\mathscr{N} \backslash \mathscr{L}$ : otherwise take $b \in N \backslash P$ from the definable closure of $A$ in $\mathscr{N}, \mathscr{N}^{\prime} \succ \mathscr{N}$ and elements $c_{1}, \ldots, c_{l} \in \mathscr{N}^{\prime}$ with $\mathscr{N}^{\prime} \models \Theta_{1}<c_{1} \in \Theta_{2}<c_{2} \in \ldots \in \Theta_{l}<c_{l}$ and $\operatorname{dim} P\left\langle b, c_{1}, \ldots, c_{l}\right\rangle=l+1$; then by (8.4), $b \in P\left\langle c_{1}, \ldots, c_{l}\right\rangle$, which is impossible.

From this, since $f$ is 0 -definable in $\mathscr{M}$ and $T_{\text {convex }, l}$ is complete, a standard compactness argument shows that there are finitely many $\mathscr{L}_{\text {convex }, l}$-formulas $\psi_{i}(\bar{x})$ and 0 -definable $\mathscr{L}$ functions $g_{1}(\bar{x}), \ldots, g_{m}(\bar{x})$ as desired.

$\underline{r-1 \rightarrow r}$. Let $\beta$ be a realization of $p_{r}$, let $V_{i}^{\prime}$ be the convex hull of $V_{i}$ in $M\langle\beta\rangle$, let $p_{i}^{\prime}$ be a coheir of $p_{i}$ on $M\langle\beta\rangle$ and let $\mathscr{M}^{\prime}:=\left(M\langle\beta\rangle, V_{1}^{\prime}, \ldots, V_{l}^{\prime}, p_{1}^{\prime}{ }^{L}, \ldots, p_{r}^{L}\right)$. By (6.8), $\mathscr{M}$ is existentially closed in $\mathscr{M}^{\prime}$. Let $\vartheta(\bar{x}, \bar{y})$ be an existential $\mathscr{L}^{*}$-formula, which defines the graph of $f$. Then

$$
\forall \bar{x}, \bar{y}_{1}, \bar{y}_{2} \vartheta\left(\bar{x}, \bar{y}_{1}\right) \wedge \vartheta\left(\bar{x}, \bar{y}_{2}\right) \rightarrow \bar{y}_{1}=\bar{y}_{2}
$$

is a universal $\mathscr{L}^{*}$-sentence valid in $\mathscr{M}$, thus valid in $\mathscr{M}^{\prime}$. Consequently the formula $\vartheta(\bar{x}, \bar{y})$ defines over $\mathscr{M}^{\prime}$ the graph of a map $f_{0}: Z \longrightarrow M\langle\beta\rangle^{k}$, where $Z=\left\{\bar{\delta} \in M\langle\beta\rangle^{n} \mid \mathscr{M}^{\prime} \models\right.$ $\exists \bar{y} \vartheta(\bar{\delta}, \bar{y})\}$. Again since $\vartheta$ is an existential formula, we have $M^{n} \subseteq Z$ and $\left.f_{0}\right|_{M^{n}}=f$. We extend $f_{0}$ to $M\langle\beta\rangle^{n}$ by $f_{0}(\bar{\delta})=0$ if $\bar{\delta} \notin Z$. Since $p_{r}^{\prime}{ }^{L}$ is $\beta$-definable in

$$
\left(M\langle\beta\rangle, V_{1}^{\prime}, \ldots, V_{l}^{\prime}, p_{1}^{\prime L}, \ldots, p_{r-1}^{L}\right),
$$

we can apply the induction hypothesis. Hence there are $\mathscr{L}^{*}$-formulas $\varphi_{1}(\bar{x}, z), \ldots, \varphi_{m}(\bar{x}, z)$ and maps $g_{1}, \ldots, g_{m}: M^{n+1} \longrightarrow M^{k}$, definable in $M$ without parameters such that

$$
\mathscr{M}^{\prime} \models \forall \bar{x} \exists ! i \in\{1, \ldots, m\} \varphi_{i}(\bar{x}, \beta) \wedge \bigwedge_{1 \leq i \leq m} \forall \bar{x} \varphi_{i}(\bar{x}, \beta) \rightarrow f_{0}(\bar{x})=g_{i}(\bar{x}, \beta) .
$$


The set defined by $\varphi_{i}(\bar{x}, \beta)$ in $M\langle\beta\rangle^{n}$ is a trace of an $\{\bar{\alpha}, \bar{\gamma}, \beta\}$-definable subset of $M\langle\bar{\alpha}, \bar{\gamma}, \beta\rangle$, where $\alpha_{i} \models V_{i}^{+}$and $\gamma_{i} \models p_{i}(i<r)$. Hence the intersection of this set with $M^{n}$ is 0 -definable in $\mathscr{M}$ by some $\mathscr{L}^{*}$-formula $\psi_{i}(\bar{x})$.

Let $N \in \mathbb{N}$ and let $C_{i, 1}^{\bar{a}}, \ldots, C_{i, N}^{\bar{a}}\left(1 \leq i \leq m, \bar{a} \in M^{n}\right)$ be the connected components of

$$
\left\{b \in M \mid g_{i}(\bar{a}, z) \text { is differentiable at } b \text { and } \frac{\partial}{\partial z} g_{i}(\bar{a}, b)=0\right\} \text {. }
$$

Let $s_{i, j}: M^{n} \longrightarrow M$ be 0-definable such that for all $\bar{a} \in M^{n}$ with $C_{i, j}^{\bar{a}} \neq \emptyset$ we have $s_{i, j}(\bar{a}) \in C_{i, j}^{\bar{a}}$. Let $g_{i, j}(\bar{x}):=g_{i}\left(\bar{x}, s_{i, j}(\bar{x})\right)$ and $\psi_{i, j}(\bar{x}):=\psi_{i}(\bar{x}) \wedge f(\bar{x})=g_{i, j}(\bar{x})$.

Let $\bar{a} \in M^{n}$. Then there is some $i$ such that $\mathscr{M} \models \psi_{i}(\bar{a})$, which is equivalent to $\mathscr{M}^{\prime} \models$ $\varphi(\bar{a}, \beta)$. We fix such an index $i$. Since $g_{i}(\bar{a}, \beta)=f_{0}(\bar{a})=f(\bar{a})=: \bar{b} \in M^{k}$ there are $c, d \in M$ with $c<\beta<d$, such that $g_{i}(\bar{a}, z)=\bar{b}$ on $(c, d)$. In particular $\frac{\partial}{\partial z} g_{i}(\bar{a}, z)$ vanishes in $(c, d)$. Take $j \in\{1, \ldots, N\}$ with $(c, d) \subseteq C_{i, j}^{\bar{a}}$. Then $g_{i}(\bar{a}, z)$ is constant on $C_{i, j}^{\bar{a}}$, which shows $g_{i, j}(\bar{a})=g_{i}\left(\bar{a}, s_{i, j}(\bar{a})\right)=\bar{b}=f(\bar{a})$, thus $\mathscr{M} \models \psi_{i, j}(\bar{a})$.

(8.6) Corollary. If an expansion $\mathscr{M}$ of $M \models T$ by convex sets defines a cut, which is not equivalent to the upper edge of a convex valuation ring, then $\mathscr{M}$ does not have definable Skolem functions.

Proof. By (7.6), we may assume that $\mathscr{M}$ is an exhaustive expansion of $M$. Suppose $\mathscr{M}$ defines a cut $p$ with $\operatorname{sign} p=0$ or $\operatorname{sign}^{*} \hat{p}=0$, say $\operatorname{sign} p=0$. Suppose there is some $f$ with $f(a)<p<a+f(a)$ for each $a>\hat{p}$. From (8.5) and weak o-minimality, we know that there is an $M$-definable map $f$ with the same property on the right hand side of $\hat{p}$. But this $f$ must fulfill $f(\hat{p})=p$ in contradiction to $\operatorname{sign} p=0$. The case $\operatorname{sign}^{*} \hat{p}=0$ is treated similarly.

(8.7) Theorem. (Curve selection lemma)

Let $M \models T$ and let $\mathscr{M}$ be an expansion of $M$ by convex subsets of $M$. If $Y \subseteq M^{r}$ is definable in $\mathscr{M}$ with parameters from $M$ and $y_{0} \in M^{r}$ is an accumulation point of $Y$, then there is a continuous map $f:(0,1] \longrightarrow Y \backslash\left\{y_{0}\right\}$, definable in $M$ with parameters from $M$, such that

$$
\lim _{t \rightarrow 0} f(t)=y_{0} .
$$

Proof. By (7.6), we may assume that $\mathscr{M}=\left(M, V_{1}, \ldots, V_{l}, G_{1}, \ldots, G_{m}, p_{1}^{L}, \ldots, p_{n}^{L}\right)$ is an exhaustive expansion of $M$ in the language $\mathscr{L}^{*}$. Moreover we may assume that $T=T^{d f}$ and $y_{0}=0$.

Claim. The theorem holds if no $p_{i}$ is dense.

To see this let $\vartheta(\bar{x})$ be an existential $\mathscr{L}^{*}$-formula, with parameters from $M$, which defines $Y$. Take $M^{\prime}=M\langle\bar{\beta}, \bar{\gamma}\rangle$ and

$$
\mathscr{M}^{\prime}=\left(M^{\prime}, W_{1}, \ldots, W_{l}, H_{1}, \ldots, H_{m}, q_{1}^{L}, \ldots, q_{n}^{L}\right)
$$

as in (8.1) and let $Y^{\prime}$ be the set defined by $\vartheta$ in $M^{\prime r}$. Since $M$ is archimedean in $M^{\prime}$, 0 is an accumulation point of $Y^{\prime}$, too. By (8.4), applied to $\left(M^{\prime}, W_{1}, \ldots, W_{l}\right)$ and weak ominimality, there is an $M$-definable map $g=\left(g_{1}, \ldots, g_{r}\right): M^{1+m+n} \longrightarrow M^{r}$, such that for all $\alpha \in(0,1] \subseteq M^{\prime}$ we have $g(\alpha, \bar{\beta}, \bar{\gamma}) \in Y^{\prime} \backslash 0$ and such that $\lim _{\alpha \rightarrow 0} g(\alpha, \bar{\beta}, \bar{\gamma})=0$. Let $\mu$ be a positive infinitely small element and let $N:=M^{\prime}\langle\mu\rangle$. Let $C_{i}$ be the convex hull of $W_{i}$ in $N$, let $E_{i}$ be the convex hull of $H_{i}$ in $N$ and let $s_{i}$ be an extension of $q_{i}$ on $N$. Then the cut $s_{i}$ is the unique extension of $q_{i}$ on $N$ and since no $p_{i}$ is dense, (7.4) implies that $\mathscr{N}:=\left(N, C_{1}, \ldots, C_{l}, E_{1}, \ldots, E_{m}, s_{1}, \ldots, s_{n}\right)$ is an elementary extension of $\mathscr{M}^{\prime}$. 
Let $\mathscr{N}_{0}$ be the restriction of $\mathscr{N}$ to $N_{0}:=M\langle\mu\rangle$ :

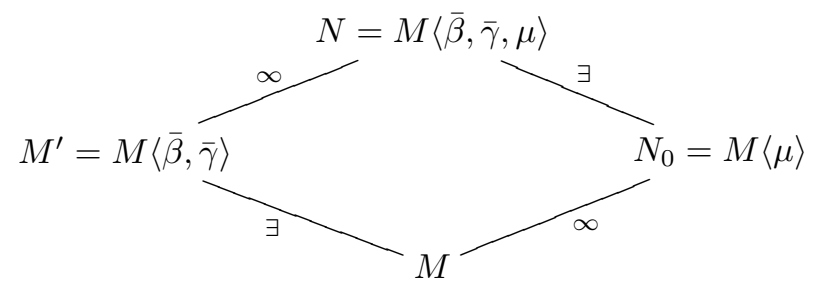

Again since no $p_{i}$ is dense, we know that $\mathscr{N}_{0}$ is an elementary extension of $\mathscr{M}$. Moreover, it is straightforward to check that the requirements of (6.8) are still true for the extension $\mathscr{N}_{0} \subseteq$ $\mathscr{N}$, hence $\mathscr{N}_{0}$ is existentially closed in $\mathscr{N}$. By o-minimality and since $M$ is archimedean in $M^{\prime}$, there are continuous $M$-definable maps $s_{1}, \ldots, s_{r}: M \longrightarrow M$ with $s_{i}(0)=0$, such that $\left|g_{i}(\mu, \bar{\beta}, \bar{\gamma})\right|<s_{i}(\mu)$. By weak o-minimality of $\mathscr{M}^{\prime}$ we have $\mathscr{N} \models \vartheta(g(\mu, \bar{\beta}, \bar{\gamma})) \wedge g(\mu, \bar{\beta}, \bar{\gamma}) \neq 0$. Since $\mathscr{N}_{0}$ is existentially closed in $\mathscr{N}$ and $\vartheta$ is an existential formula, there is an $M$-definable map $h: M \longrightarrow M^{m+n}$, such that

$$
\mathscr{N}_{0} \models \bigwedge_{1 \leq i \leq r}\left|g_{i}(\mu, h(\mu))\right|<s_{i}(\mu) \wedge \vartheta(g(\mu, h(\mu))) \wedge g(\mu, h(\mu)) \neq 0 .
$$

Let $f_{i}(x):=g_{i}(x, h(x))$ and $f:=\left(f_{1}, \ldots, f_{r}\right)$. By weak o-minimality of $\mathscr{M}$ and since $\mathscr{M} \prec \mathscr{N}_{0}$, there is some $\varepsilon \in M, \varepsilon>0$, such that $\left|f_{i}(x)\right| \leq s_{i}(x), \mathscr{M} \models \vartheta(f(x))$ and $f(x) \neq 0$ for all $x \in(0, \varepsilon)$. Since all $s_{i}$ are continuous with $s_{i}(0)=0$, the function $f$ has the desired properties on the interval $(0, \varepsilon)$. This finishes the proof of the claim.

For the general case we may assume that $\left(p_{1}, \ldots, p_{n_{0}}\right)$ is an enumeration of the dense types among the $p_{1}, \ldots, p_{n}$. Let $\mu$ be an infinitesimal element over $M$ and let $\nu$ be an infinitesimal element over $M\langle\mu\rangle$. The structures $M\langle\mu\rangle, M\langle\nu\rangle$ and $M\langle\mu, \nu\rangle$ can be expanded in a unique way to $\mathscr{L}^{*}$-structures which have $\mathscr{M}$ as a substructure and interpret the $\Theta_{i}$ as convex valuation rings, the $\mathscr{G}_{i}$ as convex subgroups and the $\mathscr{D}_{i}$ as left options of cuts. We denote these structures by $\mathscr{M}_{\mu}, \mathscr{M}_{\nu}$ and $\mathscr{M}_{\mu, \nu}$ in the sequel. If $i \in\left\{1, \ldots, n_{0}\right\}$, then the invariance group of the unique extension $p_{i, \mu}$ of $p_{i}$ on $M\langle\mu\rangle$ is the set of $M$-infinitesimal elements of $M\langle\mu\rangle$. Similar for the unique extensions $p_{i, \nu}$ and $p_{i, \mu, \nu}$ on $M\langle\nu\rangle, M\langle\mu, \nu\rangle$, respectively. Consequently if $W_{\mu}, W_{\nu}$ and $W_{\mu, \nu}$ denote the convex hulls of $M$ in $M\langle\mu\rangle, M\langle\nu\rangle$ and $M\langle\mu, \nu\rangle$ respectively, then $W_{\mu}=V\left(p_{i, \mu}\right), W_{\nu}=V\left(p_{i, \nu}\right)$ and $W_{\mu, \nu}=V\left(p_{i, \mu, \nu}\right)$ for each $i \in\left\{1, \ldots, n_{0}\right\}$. It is straightforward to check that $\left(\mathscr{M}_{\mu}, W_{\mu}\right),\left(\mathscr{M}_{\nu}, W_{\nu}\right)$ and $\left(\mathscr{M}_{\mu, \nu}, W_{\mu, \nu}\right)$ are exhaustive expansions of $M\langle\mu\rangle, M\langle\nu\rangle$ and $M\langle\mu, \nu\rangle$ respectively. Moreover, by (7.4), we have $\left(\mathscr{M}_{\mu}, W_{\mu}\right),\left(\mathscr{M}_{\nu}, W_{\nu}\right) \prec\left(\mathscr{M}_{\mu, \nu}, W_{\mu, \nu}\right)$. Now in the list of cuts of signature 0 , named in the exhaustive expansion $\left(\mathscr{M}_{\mu}, W_{\mu}\right)$, there is no dense cut anymore. By the claim, there is an $M$-definable map $g: M^{2} \longrightarrow M^{r}$, such that

$$
\left(\mathscr{M}_{\mu}, W_{\mu}\right) \models \lim _{x \rightarrow 0} g(x, \mu)=0 \wedge \forall x \in(0,1) \vartheta(g(x, \mu)) \wedge g(x, \mu) \neq 0 .
$$

Thus $\left(\mathscr{M}_{\mu, \nu}, W_{\mu, \nu}\right) \models g(\nu, \mu)=0 \wedge \vartheta(g(\nu, \mu)) \wedge g(\nu, \mu) \neq 0$. Now we proceed as in the proof of the claim: Since $M\langle\nu\rangle$ is archimedean in $M\langle\mu, \nu\rangle$ there are continuous $M$-definable maps $s_{1}, \ldots, s_{r}: M \longrightarrow M$ with $s_{i}(0)=0$, such that $\left|g_{i}(\nu, \mu)\right|<s_{i}(\nu)$. There is an $M$-definable map $h: M \longrightarrow M$, such that

$$
\left(\mathscr{M}_{\nu}, W_{\nu}\right) \models \bigwedge_{1 \leq i \leq r}\left|g_{i}(\nu, h(\nu))\right|<s_{i}(\nu) \wedge \vartheta(g(\nu, h(\nu))) \wedge g(\nu, h(\nu)) \neq 0 .
$$

Since $\vartheta$ is an $\mathscr{L}^{*}$-formula we also know that $\mathscr{M}_{\nu}$ satisfies this sentence. Let $f_{i}(x):=$ 
$g_{i}(x, h(x))$ and $f:=\left(f_{1}, \ldots, f_{r}\right)$. Since $\mathscr{M}$ is existentially closed in $\mathscr{M}_{\nu}$ the map $f$ has the desired properties.

We conclude with a description of cuts, definable in exhaustive expansions. Recall that by (7.6), every expansion of a $T$-model $M$ by a finite set of convex subsets of the line is interdefinable with some exhaustive expansion of $M$.

(8.8) TheOREM. Let $\mathscr{M}=\left(M, V_{1}, \ldots, V_{l}, G_{1}, \ldots, G_{m}, p_{1}^{L}, \ldots, p_{n}^{L}\right)$ be an exhaustive expansion of a T-model $M$ and let $r$ be a nonprincipal cut of $M$ such that $r^{L}$ is definable in $\mathscr{M}$ with parameters.

(a) $\operatorname{sign} r=0$ if and only if there is an $M$-definable map $f: M^{n} \longrightarrow M$ such that $r=$ $f\left(p_{1}, \ldots, p_{n}\right)$.

(b) $\operatorname{sign} r \neq 0$ and $\operatorname{sign}^{*} \hat{r}=0$ if and only if there are $a, b \in M$ and exponents $\lambda_{1}, \ldots, \lambda_{m}$ such that $r=a+b \cdot\left(G_{1}^{+}\right)^{\lambda_{1}} \cdot \ldots \cdot\left(G_{m}^{+}\right)^{\lambda_{n}}$.

(c) $\operatorname{sign} r \neq 0$ and $\operatorname{sign}^{*} \hat{r} \neq 0$ if and only if for some $i \in\{1, \ldots, l\}$ there are $a, b \in M$ such that $a+b \cdot V_{i}^{+}=r$ or $a+b \cdot \mathfrak{m}_{V_{i}}^{+}=r$.

Proof. The implications $\Leftarrow$ in (a) and (b) hold by (5.4). The implication $\Leftarrow$ in (c) is obvious. In order to prove the converse implications in (a), (b) and (c) we do an induction on $k:=l+m+n$. We may assume that $T=T^{d f}$ is universally axiomatized with quantifier elimination.

$\underline{k=l}$. Hence $\mathscr{M}=\left(M, V_{1}, \ldots, V_{l}\right)$. Suppose $r^{L}$ is definable in $\mathscr{M}$ with parameters. Since $\overline{T_{\text {convex }, l}}$ has quantifier elimination by $(6.3)$ and $T=T^{d f}, r^{L}$ is a boolean combination of sets of the form $\left\{f \in V_{i}\right\}$ or $\{f \geq 0\}$, where $f: M \longrightarrow M$ is $M$-definable. By weak o-minimality, this is only possible if $r$ is an edge of one of these sets. But then $r^{L}$ is already definable in $\left(M, V_{i}\right)$ for some $i \in\{1, \ldots, l\}$ and we get the theorem from (2.12).

$k \rightarrow k+1, k \geq l$. Let $k+1=l+m+n>l$. Suppose one of the conditions (a)-(c) does not hold. Then (7.2) together with (5.4) implies that $\left(V_{1}^{+}, \ldots, V_{l}^{+}, G_{1}^{+}, \ldots, G_{m}^{+}, p_{1}, \ldots, p_{n}, r\right)$ is a box type. By model completeness of exhaustive expansions (cf. (7.4)) there is an existential $\mathscr{L}\left(\Theta_{1}, \ldots, \Theta_{l}, \mathcal{G}_{1}, \ldots, \mathcal{G}_{m}, \mathcal{D}_{1}, \ldots, \mathcal{D}_{n}\right)$-formula $\psi(x)$ with parameters from $M$, such that $r^{L}$ is defined by $\psi(x)$ in $\mathscr{M}$. We write

$$
\left(q_{1}, \ldots, q_{k}, q\right)=\left(V_{1}^{+}, \ldots, V_{l}^{+}, G_{1}^{+}, \ldots, G_{m}^{+}, p_{1}, \ldots, p_{n}\right)
$$

Let $\alpha$ be a realization of $q$ and let $N:=M\langle\alpha\rangle$. Let $q_{1}^{\prime}, \ldots, q_{k}^{\prime}, r^{\prime}$ be the unique extensions of $q_{1}, \ldots, q_{k}, r$ on $N$, respectively and let $q^{\prime}$ be a coheir of $q$ on $N$. Let $\mathscr{N}:=\left(N, q_{1}^{L}, \ldots, q_{k}^{\prime L}, q^{L}\right)$. Since $\mathscr{M}$ is exhaustive, (6.8) says that $\mathscr{M}$ is existentially closed in $\mathscr{N}$. Since $\psi$ is an existential formula, the set defined by $\psi$ in $\mathscr{N}$ intersects $M$ in $r^{L}$. Hence the upper edge of this set is $r^{\prime}$, i.e. $r^{\prime L}$ is definable in $\mathscr{N}$.

Since $k \geq l$ and $\mathscr{M}$ is exhaustive, either $k \geq l+m$ and $\operatorname{sign} q=0$ or $k<l+m$ and $q=\hat{q}$, $\operatorname{sign}^{*} \hat{q}=0$. It follows $k \geq l+m$ and $\operatorname{sign} q^{\prime} \neq 0$ (by (2.4)), or $k<l+m$ and $q^{\prime}=\hat{q^{\prime}}$, $\operatorname{sign}^{*} \hat{q}^{\prime} \neq 0$ (by (2.4) applied multiplicatively). Thus $k \geq l+m$ and $q^{L}$ is definable in $\left(N, G\left(q^{\prime}\right)\right)$ or $k<l+m$ and $q^{L}$ is definable in $\left(N, V\left(q^{\prime}\right)\right)$. Let $\mathscr{N}_{0}:=\left(N, q_{1}^{\prime L}, \ldots, q_{k}^{\prime L}\right)$. Since $\mathscr{M}$ is exhaustive, we have $k \geq l+m$ and $G\left(q^{\prime}\right)$ is definable in $\mathscr{N}_{0}$ or $k<l+m$ and $V\left(q^{\prime}\right)$ is definable in $\mathscr{N}_{0}$; the argument is the same as at the end of the proof of (7.5). In each case it follows that $q^{L}$ is definable in $\mathscr{N}_{0}$ with parameters.

Since $r^{L}$ is definable in $\mathscr{N}$ it follows that $r^{L}$ is definable in $\mathscr{N}_{0}$ with parameters. By (7.5), $\mathscr{N}_{0}$ is exhaustive and from the induction hypothesis we get that $\left(q_{1}^{\prime}, \ldots, q_{k}^{\prime}, r^{\prime}\right)$ is not a box type. This contradicts (3.4) and our assumption that $\left(q_{1}, \ldots, q_{k}, r, q\right)$ is a box type. 
(8.9) Corollary. If $p, q$ are nonprincipal cuts of a T-model $M$ and $q^{L}$ is definable in $\left(M, p^{L}\right)$, then $q \sim p, q \sim \hat{p}$ or $q \sim V(p)^{+}$.

Proof. By (7.6) and (8.8).

Acknowledgements. I wish to thank Thomas Güldenberg for many helpful comments on an earlier version of the manuscript.

\section{REFERENCES}

[Ba-Po] Y. Baisalov, B. Poizat; Paires de Structures o-minimales; J. Symbolic Logic 63 (1998), no. 2, 570-578

[vdD-Lew] L. van den Dries, A.H. Lewenberg; T-convexity and tame extensions; J. Symbolic Logic 60 (1995), no. 1, 74-102

[vdD-Sp] L. van den Dries, P. Speissegger; The field of reals with multisummable series and the exponential function; Proc. London Math. Soc. (3) 81 (2000), no. 3, $513-565$.

[Do] A. Dolich; Forking and independence in o-minimal theories; J. Symbolic Logic 69 (2004), no. 1, 215-240

[Kn] M. Knebusch; Weakly semialgebraic spaces; Lecture Notes in Mathematics, 1367, Springer-Verlag, Berlin, 1989

[La-Po] D. Lascar, B. Poizat; An Introduction to Forking; J. Symbolic Logic 44 (1979), no. $3,330-350$

[La] D. Lascar; Stability in Model Theory; Pitman Monographs and Surveys in Pure and Applied Mathematics, 36. Longman Scientific \& Technical, Harlow; John Wiley \& Sons, Inc., New York, 1987

[MMS] D. Macpherson, D. Marker, C. Steinhorn; Weakly o-minimal structures and real closed fields; Trans. Amer. Math. Soc. 352 (2000), no. 12, 5435-5483

[Ma] D. Marker; Omitting types in o-minimal theories; J. Symbolic Logic 51 (1986), no. $1,63-74$

[Ma-St] D. Marker, C. Steinhorn; Definable types in o-minimal theories; J. Symbolic Logic 59 (1994), 185-198

[PS] A. Pillay, C. Steinhorn; Definable sets in Ordered Structures I; Bull. Amer. Math. Soc. (N.S.) 11 (1984), no. 1, 159-162

[Poi] Poizat, B.; Cours de théorie des modèles; Villeurbanne: Nur al-Mantiq walMa'rifah (1985)

[She1] S. Shelah; Classification theory and the number of non-isomorphic models; Studies in logic and the foundations of mathematics, North-Holland, Amsterdam, 1978

[She2] S. Shelah; Dependent first order theories, continued; SH783

[Sho] J. R. Shoenfield; Mathematical logic; Addison-Wesley Publishing Co., Reading, Mass.-London-Don Mills, Ont. 1967

[Tr1] M. Tressl; Model Completeness of o-minimal Structures expanded by Dedekind Cuts; J. Symbolic Logic 70 (2005), no. 1, 29-60

[Tr2] M. Tressl; The elementary theory of Dedekind cuts in polynomially bounded structures; Ann. Pure Appl. Logic 135 (2005), no. 1-3, 113-134

[Tr3] M. Tressl; Pseudo Completions and Completions in Stages of o-minimal structures; Arch. Math. Logic (2006), vol 45, no. 8, 983-1009

[Tr4] M. Tressl; Valuation theoretic content of the Marker-Steinhorn Theorem; J. Symbolic Logic 69 (2004), no. 1, 91-93

[We1] R. Wencel; Weakly o-minimal non-valuational structures; preprint 2005 
[We2] R. Wencel; Topological properties of sets definable in weakly o-minimal structures; preprint 2005

University of Manchester, School of Mathematics, Manchester M13 9PL, UK e-mail: marcus.tressl@manchester.ac.uk 